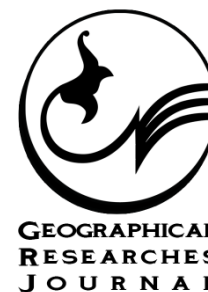

\title{
Relationship of Drought and Teleconnection Patterns; Case Study of Qara-Qom Basin
}

\section{A R T I C LE I N F O}

\section{Article Type}

Original Research

\section{Authors}

Fallahzadeh M. ${ }^{1} M A$,

Rezaei P. ${ }^{* 1} P h D$,

Eslamian S. ${ }^{2} P h D$,

Abbasi A. ${ }^{1} P h D$

\section{How to cite this article} Fallahzadeh M, Rezaei P, Eslamian S, Abbasi A. Relationship of Drought and Teleconnection Patterns; Case Study of Qara-Qom Basin. Geographical Researches Quarterly Journal. 2019;34(2): 153-164.
${ }^{1}$ Department of Geography, Faculty of Humanities, Najaf Abad Branch, Islamic Azad University, Najaf Abad, Iran

${ }^{2}$ Department of Water Engineering, Faculty of Agriculture, Isfahan University of Technology, Isfahan, Iran

\section{*Correspondence}

Address: Department of Geography, Islamic Azad University, Rasht, IranPhone: +98 (51) 37136793

Fax: -

rezaei@iaurasht.ac.ir

\section{Article History}

Received: August 20, 2018

Accepted: January 01, 2019

ePublished: June 20, 2019

\section{A B S T R A C T}

Aims \& Backgrounds Drought is a natural feature of an area and its occurrence in each region can lead to economic, social and environmental losses. The aim of the present study was to investigate the relationship between the teleconnection patterns with drought in the Qara-Qom basin.

Methodology The precipitation data of 30 rain gauge and synoptic stations and the data of 32 numerical indexes of teleconnection from NOAA site were obtained during 1987-2013. The data of standardized precipitation index in the 9 to 48-month scale were classified by factor analysis and the stations with similar behavior were identified in the study area. Then, the relationship between the average drought index of each area with each of the teleconnection patterns was evaluated simultaneously with a delay of 6 and 9 months through the correlation statistical method.

Findings The multivariate ENSO index, the decadal oscillation of Pacific ocean, southern oscillation, Nino $4,3 / 4$ and 3 had the most significant correlation with the standardized precipitation index scales. Also, there was a direct correlation between the 6-month delayed drought index (With four factors of drought index) with Nino 4, 3/4 and 3, multivariate ENSO, Madden Julian oscillation in the regions of $20^{\circ}, 70^{\circ}, 80^{\circ}, 100^{\circ}$ east, and there was a significant inverse correlation between the 6-month delayed drought index with the east pacific index, the southern and Madden Julian oscillations in the regions of $160^{\circ}$ East, $120^{\circ}$ and $40^{\circ}$ West. Four factors of the 9-month delayed drought index (total basin stations) had a significant inverse correlation with the east Pacific index, southern and Madden Julian oscillations in the regions of $120^{\circ}$ and $40^{\circ}$ West, and it had a significant direct relationship with the index of Nino 3 , the multivariate ENSO and the oscillation of Madden Julian in the regions of $70^{\circ}, 80^{\circ}$ and $100^{\circ}$ East. Conclusion There is a relationship between the teleconnection patterns and droughts in Qara-Qom basin.

Keywords Standardized Precipitation Index (SPI); Teleconnection; Factor Analysis; Correlation; Qara Qom Basin

\section{I T A T I O N L I N K S}

[Ilderumi, et al; 2017] Study of Relation between ENSO phenomenon and drought occurrence [Babaiean, et al; 2013] Investigating the behavior of Qara Qom Basin water resources under the ... [Khosravi;2004] A Survey on the relations of the northern hemisphere large scale circulation patterns ...; [Khorshiddost, et al; 2010] Application of large-scale atmospheric-oceanographic patterns in the ...; [Darand; 2015] Drought monitoring in Iran by Palmer Severity Drought Index [PDSI] and ...; [Zolfaghari \& Nouri Sameleh; 2016] Determination of suitable variables for analysis of droughts in Iran by using ...; [Sadatinejad, et al; 2016] Frequency analysis of regional meteorological drought in Karun-1 ...; [Shafiei \& Raeini; 2016] Assessment of the impact of meteorological drought and wetness trends on the ... [Shafiee \& Ghahraman; 2009] Investigation of place variations of maximum probable 24-hour ...; [Alizadeh, et al; 2011] Investigating the effect of teleconnection patterns on parameters of precipitation ...; [Fatehi Marj; 2015] Study of the relationship between the some climate signals ...; [Ghavidel Rahimi, et al; 2014] Investigation on North Sea-Caspian teleconnection pattern effect on autumn rainfall ...; [Ghavidel Rahimi, et al; 2014] The role of a teleconnection pattern the far atmosphere of the North SeaMazandaran Sea in the changes in the rainfall of the ...; [Mahdavi \& Taherkhani; 2012] Application of statistics in ...; [Nayebi; 2015] Advanced applied statistics with ...; [Asong, et al; 2018] Historical drought patterns over Canada and their teleconnections with large-scale ...; [Baek, et al; 2017] Precipitation, temperature, and teleconnection signals across the combined North American ...; [Chowdhury, 2003] The El Nino- Southern Oscillation [ENSO] and seasonal flooding ...; [Hendon; 2003] Indonesian rainfall variability: Impacts of ENSO and local air-sea ...; [Lee HF; 2011] Relationship between NAO and drought disasters in northwestern China ...; [Oglesby, et al; 2012] The role of the Atlantic multidecadal oscillation on medieval drought in North America synthesizing results from proxy data ...; [Rajagopalan, et al; 2000] Spatiotemporal variability of ENSO and SST teleconnections to summer drought over the United ...; [Wang \& Kumar; 2015] Assessing the impact of ENSO on drought in the U.S ... 
كلانى بر جامعه و محيطزيست وارد سازد، جنانجِه در سالهاى اخير

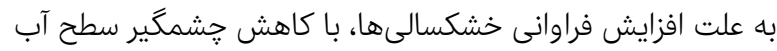

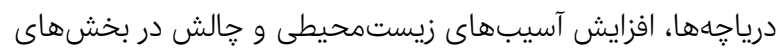

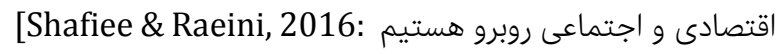

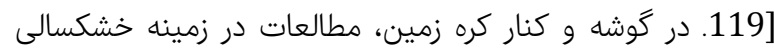

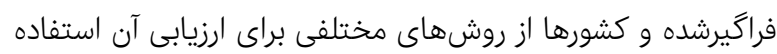

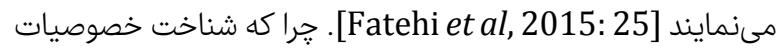

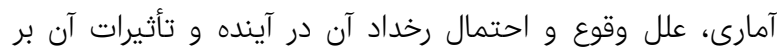
حوزمهاى مختلف از اهم مطالعات جغرافيايى در مناطق مختلف

است] [Sadatinejad et al, 2016: 108] تاكنون محققين توانستهاند با استفاده از تغييرات ميدانهاي فإن فشار

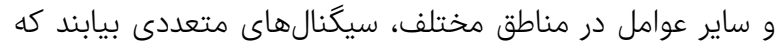

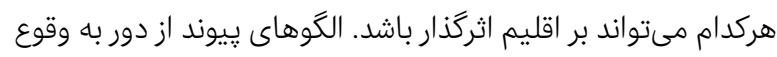

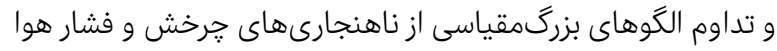

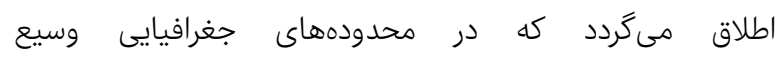

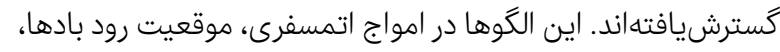

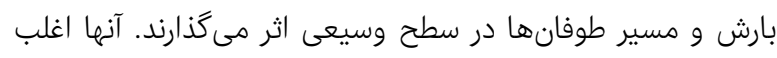

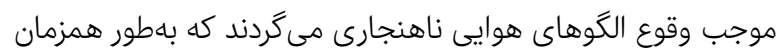

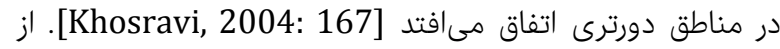

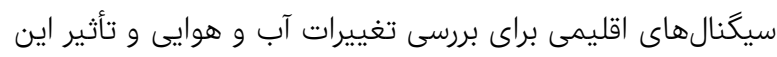

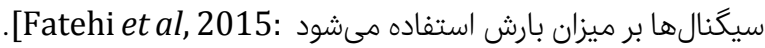

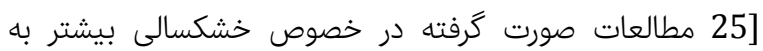

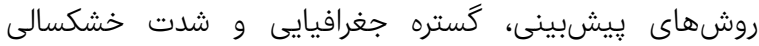
معطوف بوده است. لذا مطالعه ارتباط رفتارى خشكسالى بائ

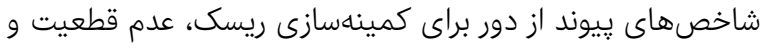
خسارتهاى ناشى از آن ضرورت دارد. درائ در اين ميان آثار بِيوند از دور

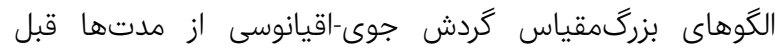

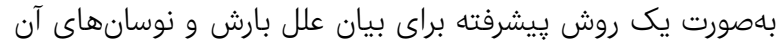

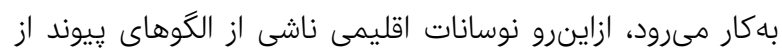

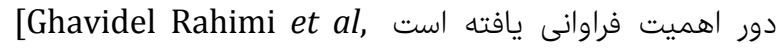
קنانجه در بررسى النينو-نوسان جنوبى و سيلاب فصلى بنكَلادش،

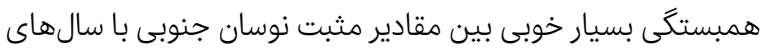
تر و مقادير منفى آن با سالهاى خشكى در اقليم بنكلادش

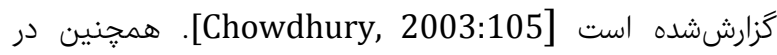

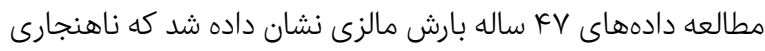

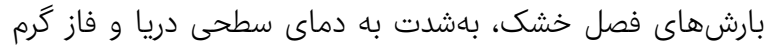

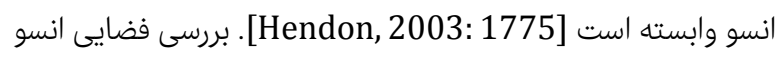
و تله كانكشنهاى سطح دريا نشان داد كه شاخص انسو نقش بسيار زيادى بر خشكسالىهاى تابستانى قرن بيستم ايالاتمتحده داريان دارد

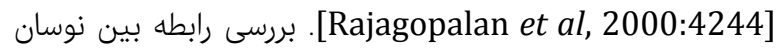

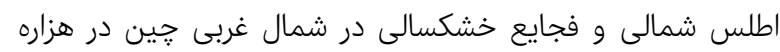
كذشته نشان داد كه نوسان اطلس شمالى و فاجعه خشكسالى دارئ دارى همبستگى منفى هستند [Lee et al, 2011: 1114]. در بررسى
ارتباط الكوهاى يّيوند از دور با خشكسالى؛ مطالعه موردى حوضه قرهقوم

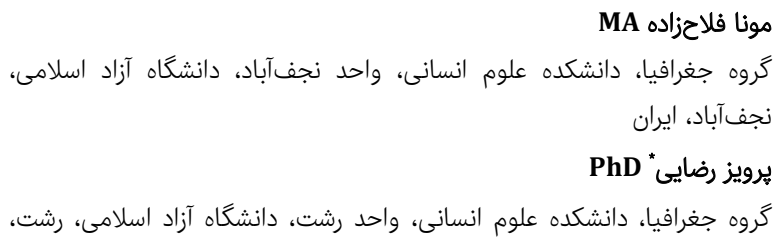
ايران

سيدسعيد اسلاميان PhD گروه مهندسى آب، دانشكده كشاورزى، دانشعاه صنعتى اصفهان، اصفهان، ايران

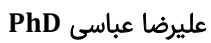
گروه جغرافيا، دانشكده علوم انسانى، واحد نجفآباد، دانشگاه آزاد اسلامى، نجفآباد، ايران

جكيده اهداف و زمينهها: خشكسالى از ويزگىهاى طبيعى است و در هر منطقهاى كه

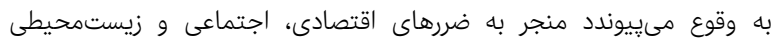

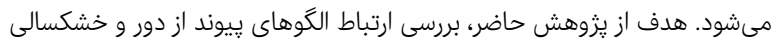
در حوضه قرهقوم بود.

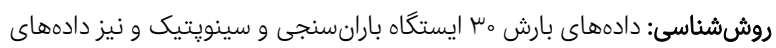

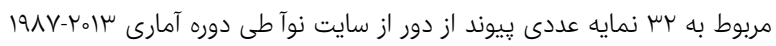

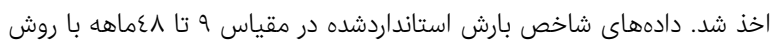

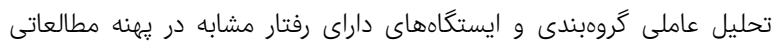

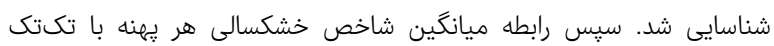

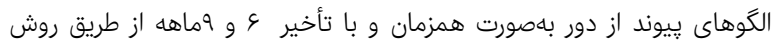
آمارى همبستخى ارزيابى شد. يافتهها: الكوى جندمتغيره انسو نوسان دههاى اقيانوس آرام، نوسان جنوبى، نينو

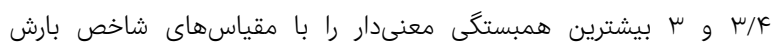

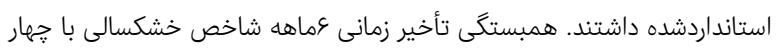

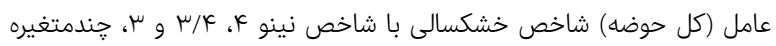

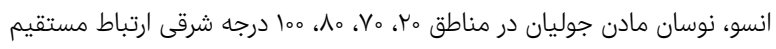

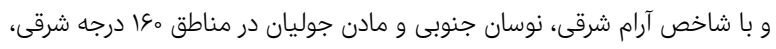

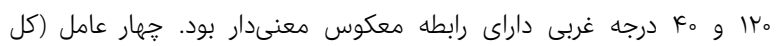

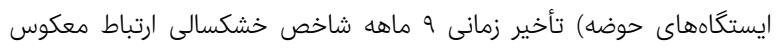

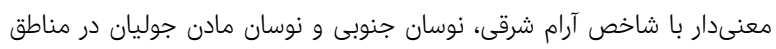

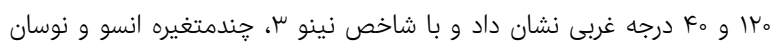

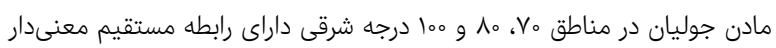

داشت. نتيجه گيرى: ارتباط معنىدارى بين الگوهاى يِيوند از دور با خشكسالىهاى حوضه قرمقوم وجود دارد.

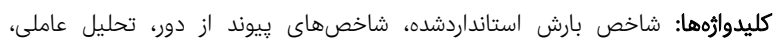
همبستخى، حوضه قرهقوم

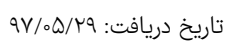

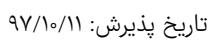

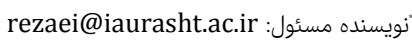

\section{مقدمه}

خشكسالى از يديدههاى زيان آور طبيعى است كه انسان همواره با

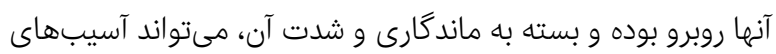


ايستكاههاى منتخب با الكوى بِيوند از دور درياى شمال-خزر وجود

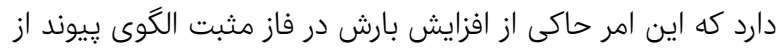

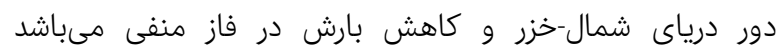

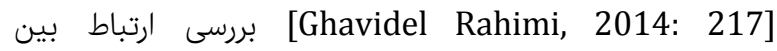
سيكنالهاى اقليمى نوسان جنوبى، جند متغيره انسو، نينو، نوسان إنيان

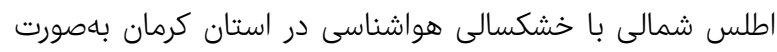

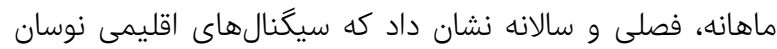

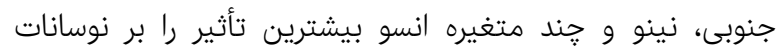
بارندگى در استان كرمان دارند[Fatehi et al, 2015:25] در

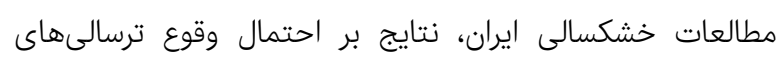

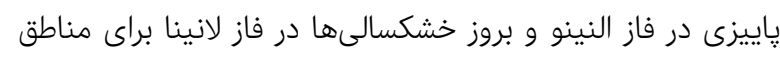

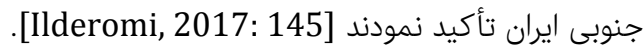

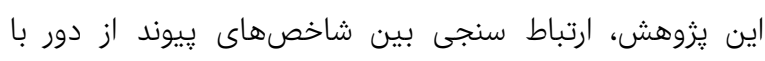

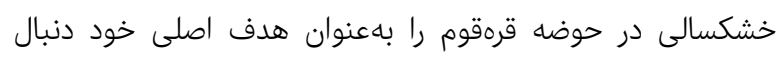

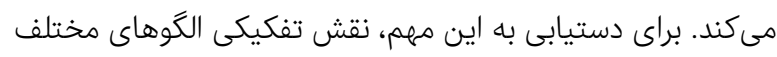

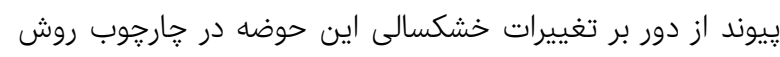

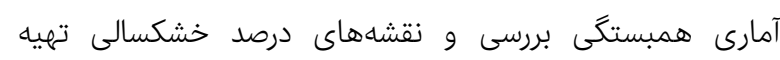

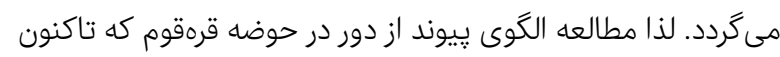

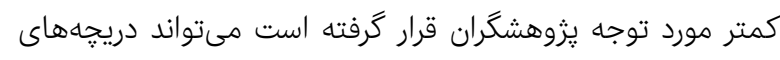

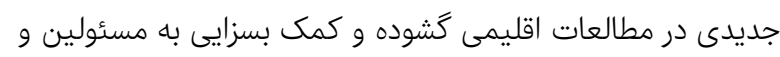

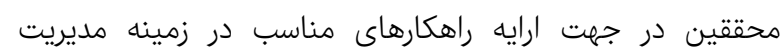

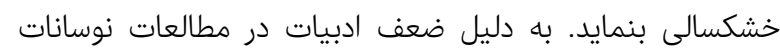

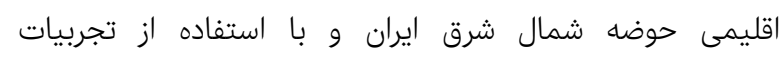

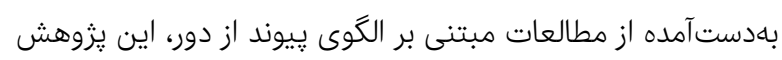

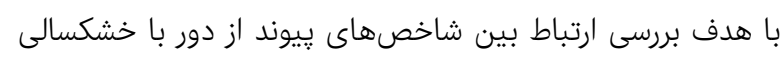

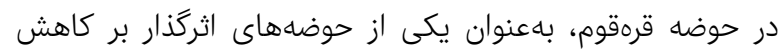

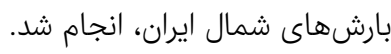

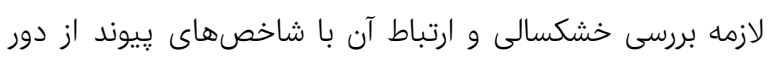

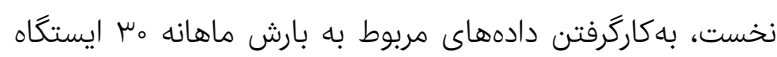

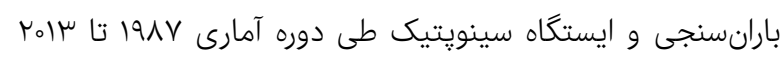

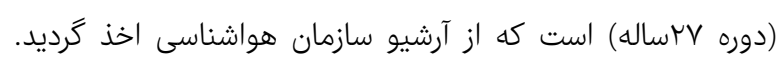

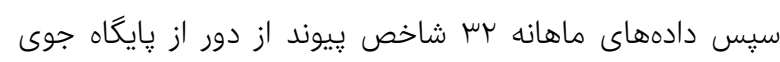

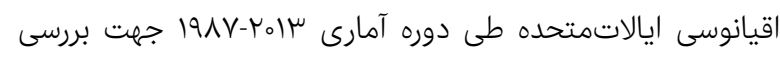

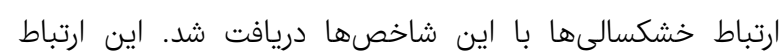

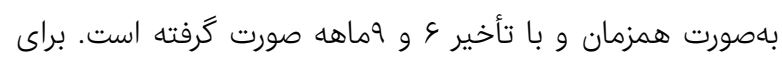

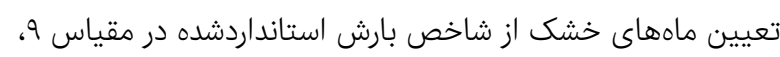

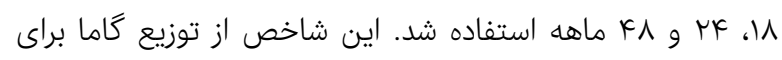

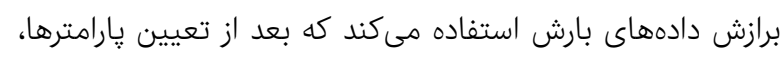

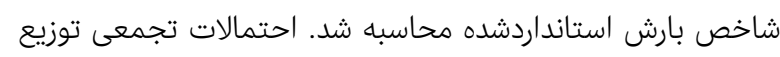

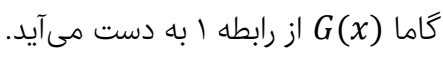

$G(x)=\frac{1}{\beta^{x} \Gamma(\alpha)} \int_{1}^{x} x^{\alpha-1} \varepsilon^{\frac{-x}{\beta}}$
نقش نوسان קند دهاء اقيانوس اطلس بر خشكسالى قرون وسطى إنى

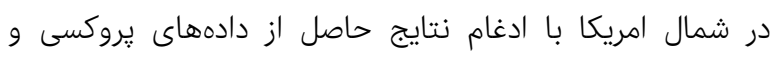

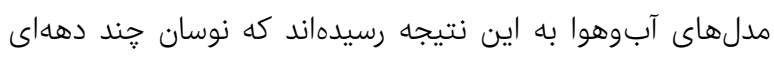

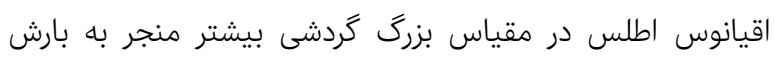

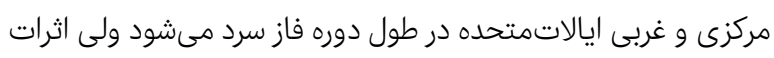

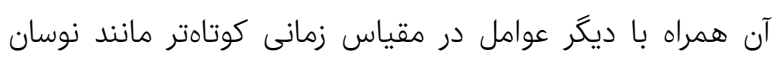

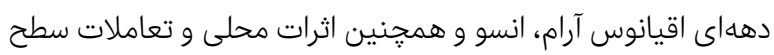

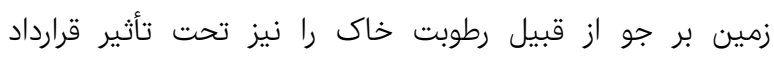

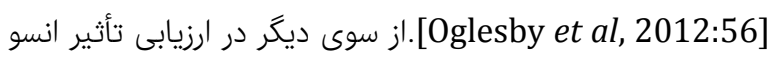

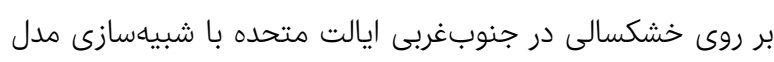

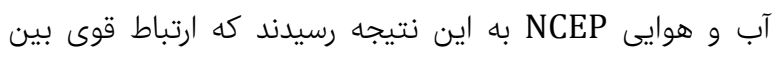

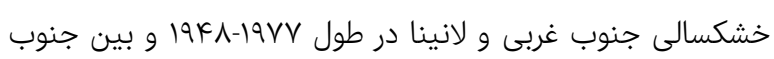

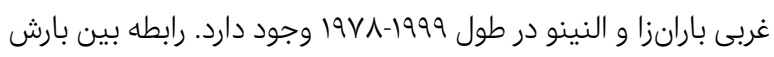

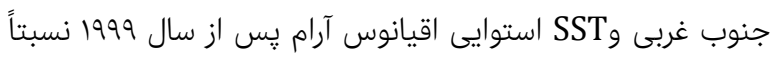

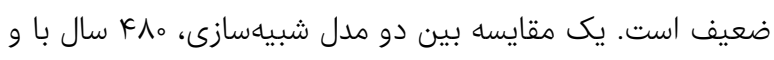

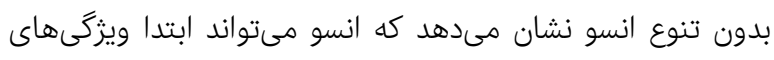

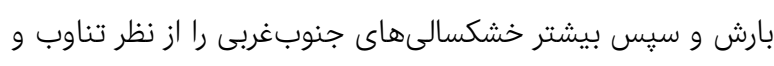

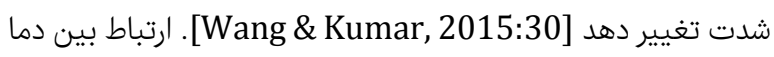

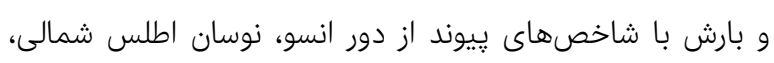

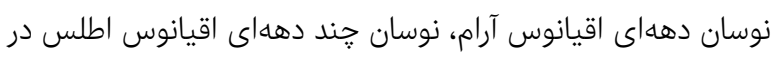

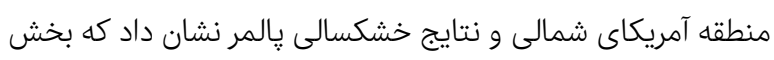

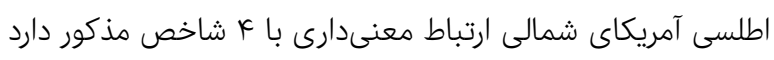

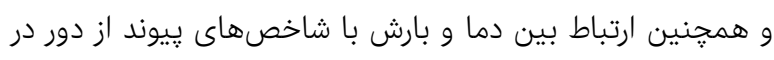

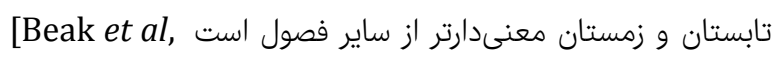

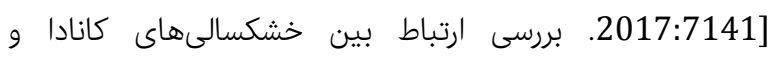

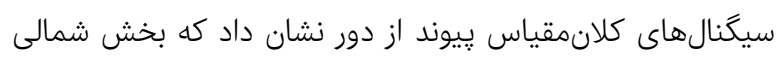

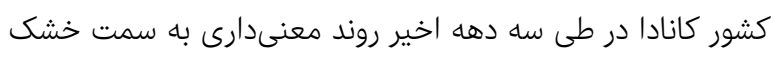

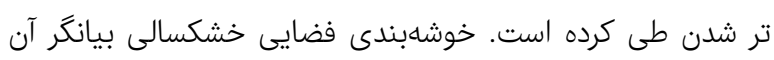

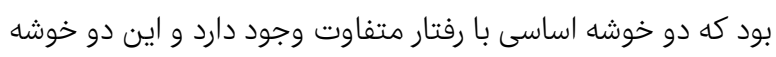

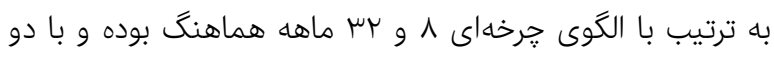

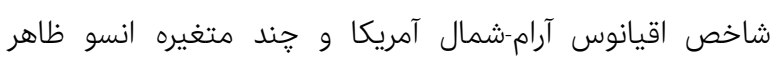

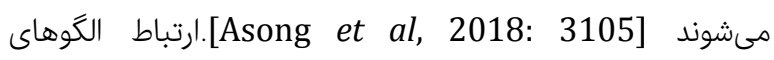

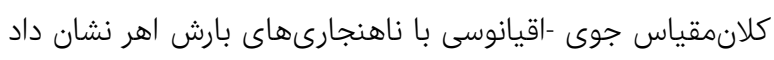

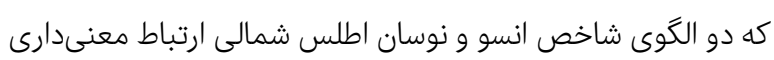

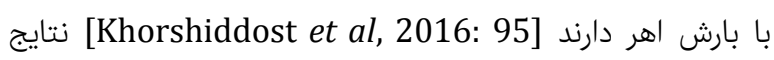

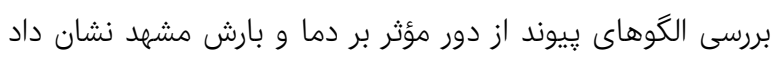

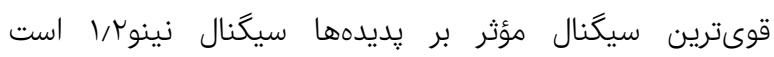

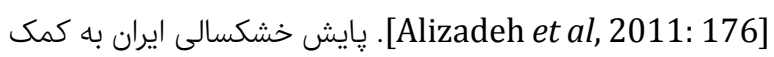

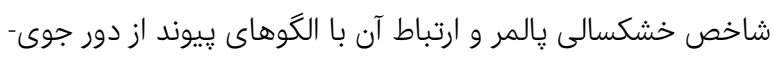

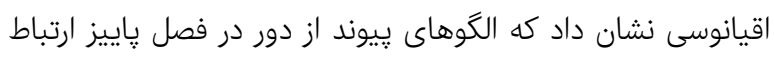

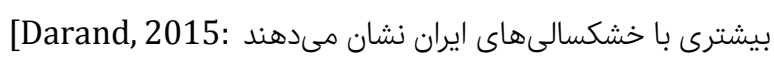

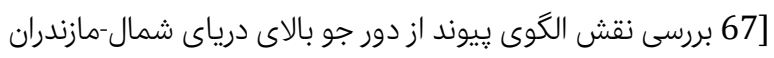

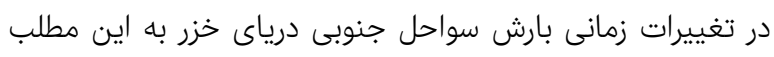

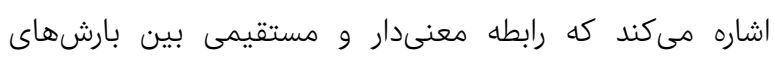


بعد از محاسبه شاخص خشكسالى ايستكاههاى حوضه قرهقوم،

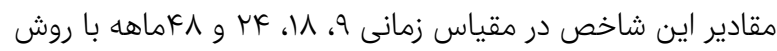

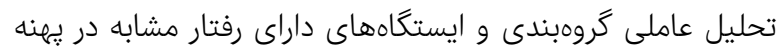

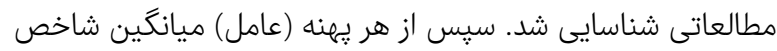

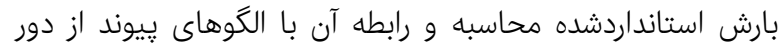

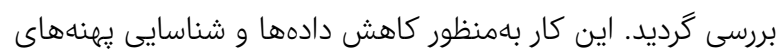

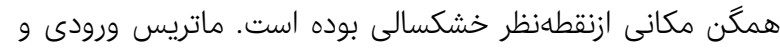

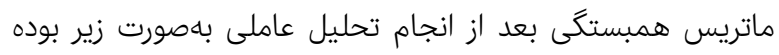
است.

$R_{\text {input }}=\begin{array}{cccc}v_{1} & v_{2} & \ldots & v_{30} \\ c_{1} & c_{2} \\ \vdots & & & \\ c_{n}\end{array}\left[\begin{array}{lll} \\ \end{array}\right.$

$R_{\text {correlation }}=\begin{gathered}v_{1} \\ v_{2} \\ \vdots \\ v_{30}\end{gathered}\left[\begin{array}{llll} & v_{2} & \ldots & v_{30} \\ & & & \\ & & & \end{array}\right]$

در اينجا v و C به ترتيب بيانگر ايستخاه و زمان هستند. همان گُنه كه مشاهده مىشود سطرهاى ماتريس، دادههاى ورودى مقائ مقادير

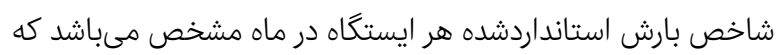

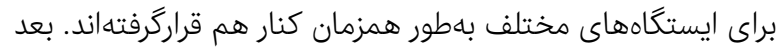

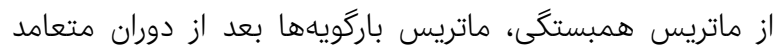

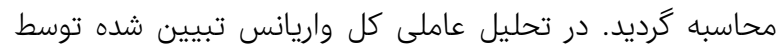

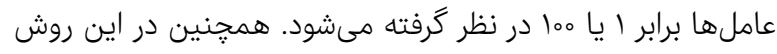

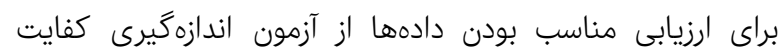
$K M O=\frac{\sum_{i} \Sigma_{j} r_{i j}^{2}}{\sum_{i} \Sigma_{j} r_{i j}^{2}+\Sigma_{i} \Sigma_{j} q_{i j}^{2}}$

كه در آن، rij مجذور همبستگى ساده ييرسون بين دو متغير فو x است و $x_{j}$

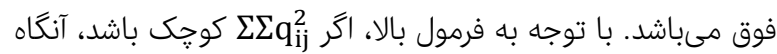

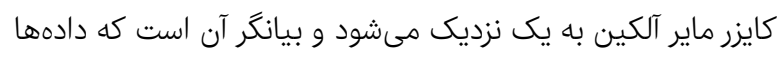

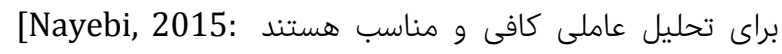

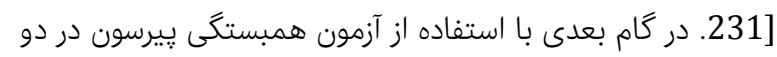

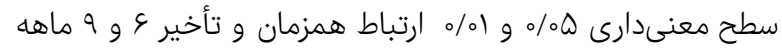

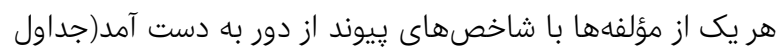

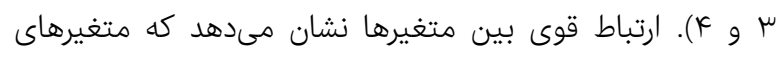

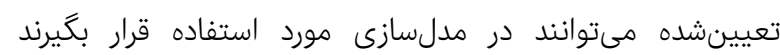

[Zolfaghari \& Nouri Sameleh, 2016: 103]

$r=\frac{\sum_{i=1}^{n}\left(X_{i}-\bar{X}\right)\left(Y_{i}-\bar{Y}\right)}{\sqrt{\sum_{i=1}^{n}\left(X_{i}-\bar{X}\right)^{2}} \sqrt{\sum_{i=1}^{n}\left(Y_{i}-\bar{Y}\right)^{2}}}$

(1)
كه در اين معادله $\alpha$ و $\beta$ به ترتيب مقدار پارامتر شكل و مقياس، $x$ مقار

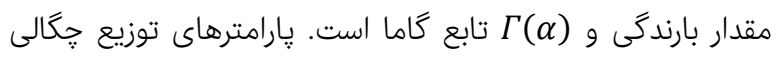

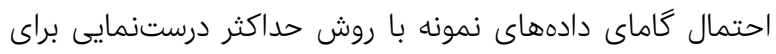

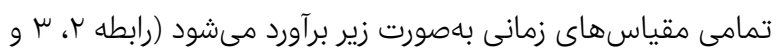

$\tilde{\alpha}=\frac{1}{4 A}[1+\hat{\beta} \sqrt{1 \pm(4 A / \alpha)}]$

$\hat{\beta}=X / \tilde{\alpha}$

$A=\ln (x)=\frac{\sum \ln (x)}{n}$

كه n تعداد مشاهدات بارندگى و X ميانكين بارندگى در طى دوره

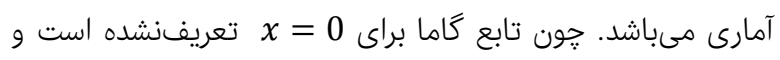

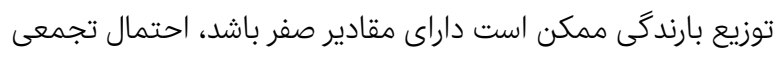

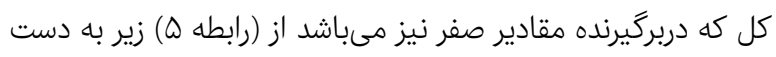
مى آيد.

$F(x)=q+(1-q) G(x)$

كه q احتمال صفر بودن مقدار بارندگى و $q$ و درنهايت با جابجايى احتمال توزيع تجمعى كاما به توزيع نرمال،

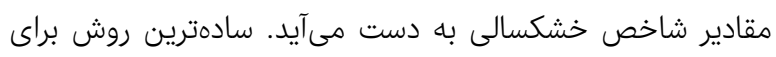

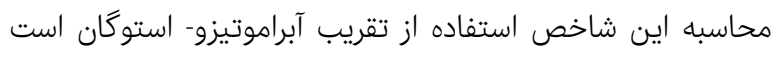

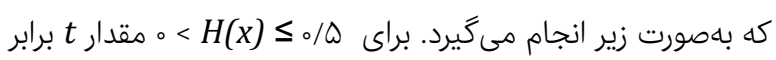

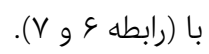

$t=\sqrt{\ln \frac{1}{(H(x))^{2}}}$ و براى اك

$t=\sqrt{\ln \left[\frac{1}{(1-H(x))^{2}}\right]}$

خواهد بود. درنهايت مقادير جابهجاشده گاما با استفاده از فرمولهاى

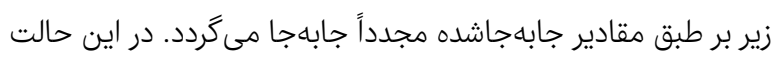

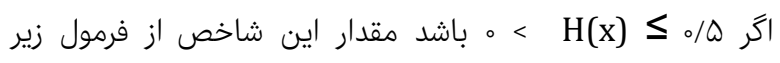
قابلمحاسبه است.

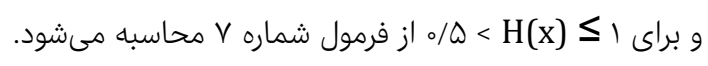
$S P I=+\left[t-\frac{c_{0}+c_{1} t+c_{2} t^{2}}{1+d_{1} t+d_{2} t^{2}+d_{3} t^{3}}\right]$ 


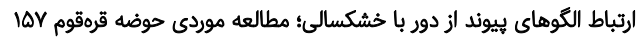

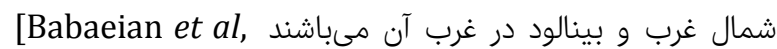

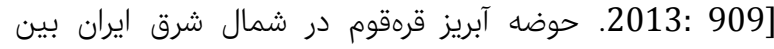
عرض هاى" واقعشده است و بخشى از حوضه بزرگ قرهقوم و قزل قوم است كه

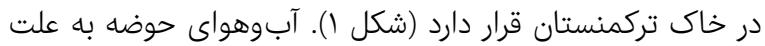

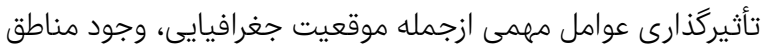

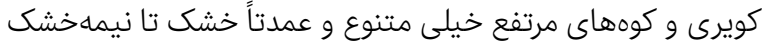

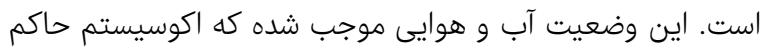
بر مناطق بيابانى حوضه از يك شرايط حساس و شكننده برخوردار

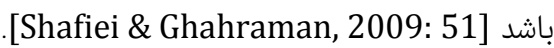

ميزان ضريب همبستگى بين ا+ تا ا- متغير است Mahdavi \&]. [ معمولاً رابطه بين دو يا جند متغير Taherkhani, 2012: 158] ازآنجهت موردمطالعه قرار مى گيرد كه از وجود رابطه بين متغيرها

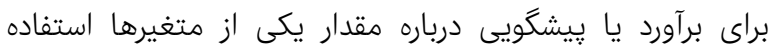

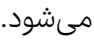
حوضه آبريز قرهقوم يكى از مس حوضه بزرگ كشـور اسـت كـه در

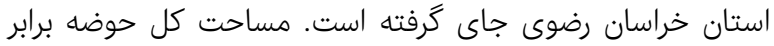

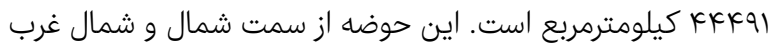
هممرز با تركمنستان و از سمت شرق بـا افغانستان همرمرز مى بـاشد. مهمترين رشتهكوههاى اين حوضه شامل هزار مسجد در شمال و

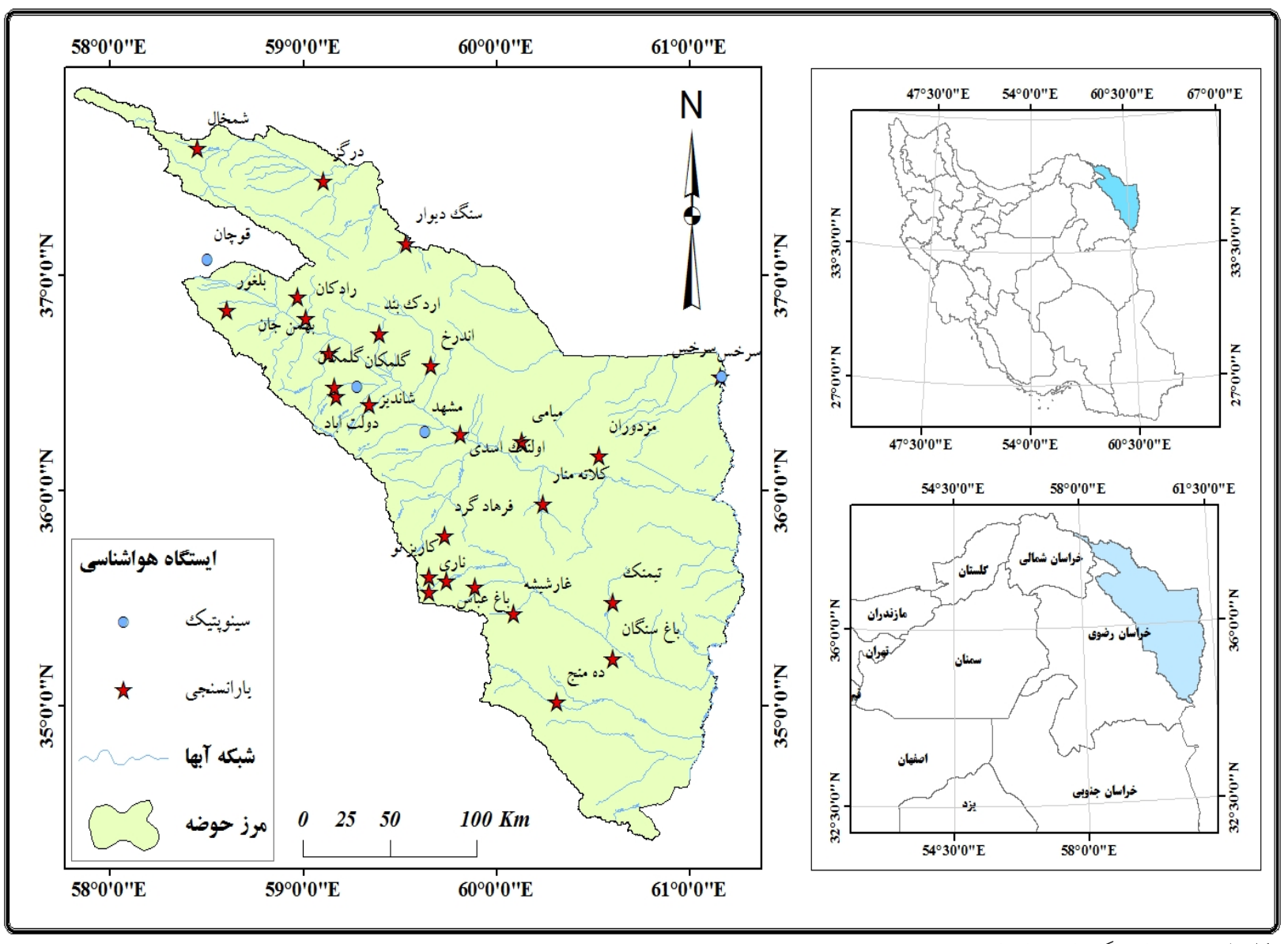

شكل () موقعيت ايستكاههاى هواشناسى درحوضه قره قوم

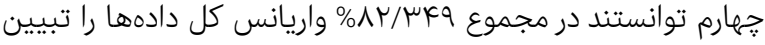

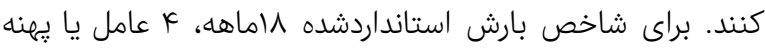
همگن تعيين شد كه عاملهاى اول تا جهارم توانستند در مجموع بارسن

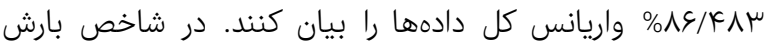

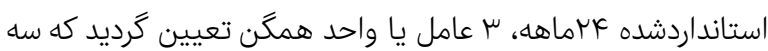

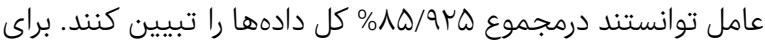

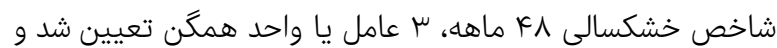

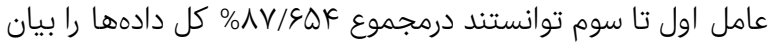
كنند. با توجه به نمودار سنكريزه، تحليل عاملى انجام شد (شكل r ب).
يافتهها و بحث

جهت بررسى ارتباط شاخص خشكسالى حوضه قرهقوم در بازههاى

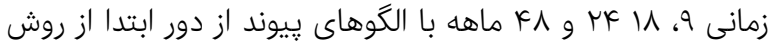

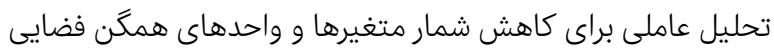

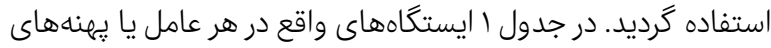

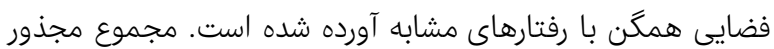

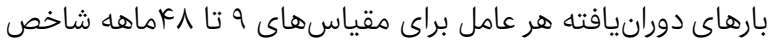

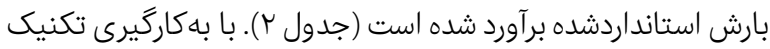

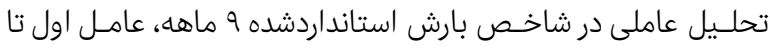




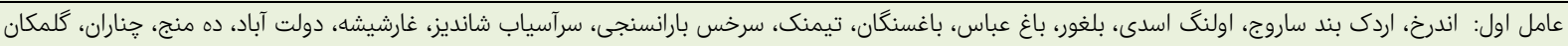

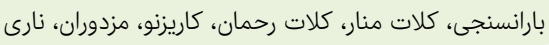

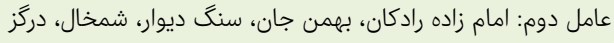

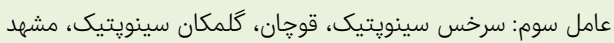

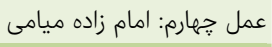

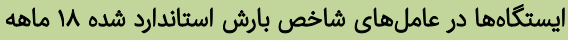

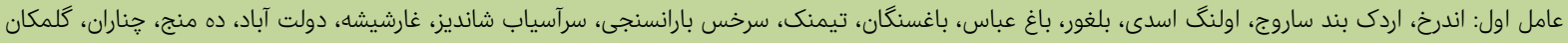

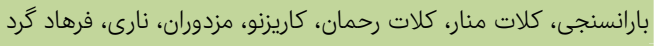

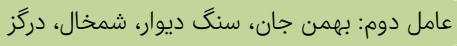

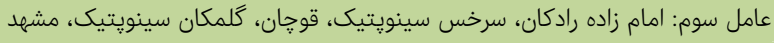

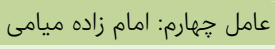

ايستگاهها در عاملهاى شاخص بارش: امام زاده ميامى استاندارد شده عr ماهه

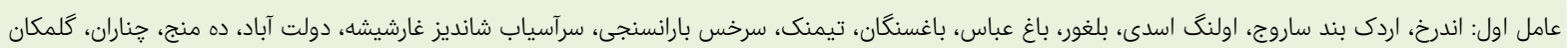

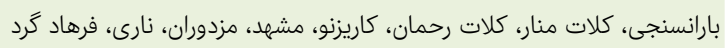

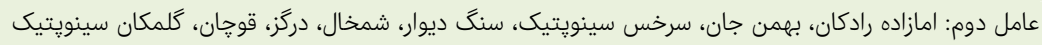
عامل سوم: امام زاده ميامى دادم:

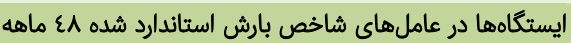

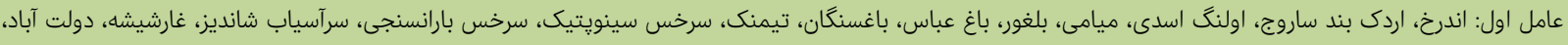

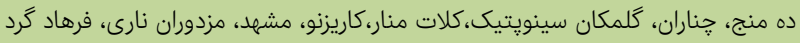

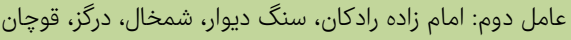

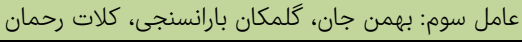

جدول r) مجموع مجذور بارهاى دورانيافته در عاملهاى شاخص خشكسالى 9 تا مع ماهه

\begin{tabular}{|c|c|c|c|c|}
\hline FاهA & & 1اماهه & وماهه & عامل \\
\hline$\Delta 1 / 9 \Delta 9$ & $\varepsilon V / .0 \Lambda$ & $\varepsilon r / \circ q r$ & $k_{0} / l_{0} \Lambda$ & اول \\
\hline VI/GYG & $V \mu / 77 r$ & $7 r / 07 \pi$ & $O \wedge / \wedge \varepsilon \vee$ & دوم \\
\hline$\Lambda V / \& \Delta F$ & No/9ro & Vo/70。 & $V Y / V \cdot \Lambda$ & سوم \\
\hline- & - & $\wedge \tau / \varepsilon \wedge \mu$ & $\wedge r / \mu \varepsilon q$ & جهارم \\
\hline
\end{tabular}
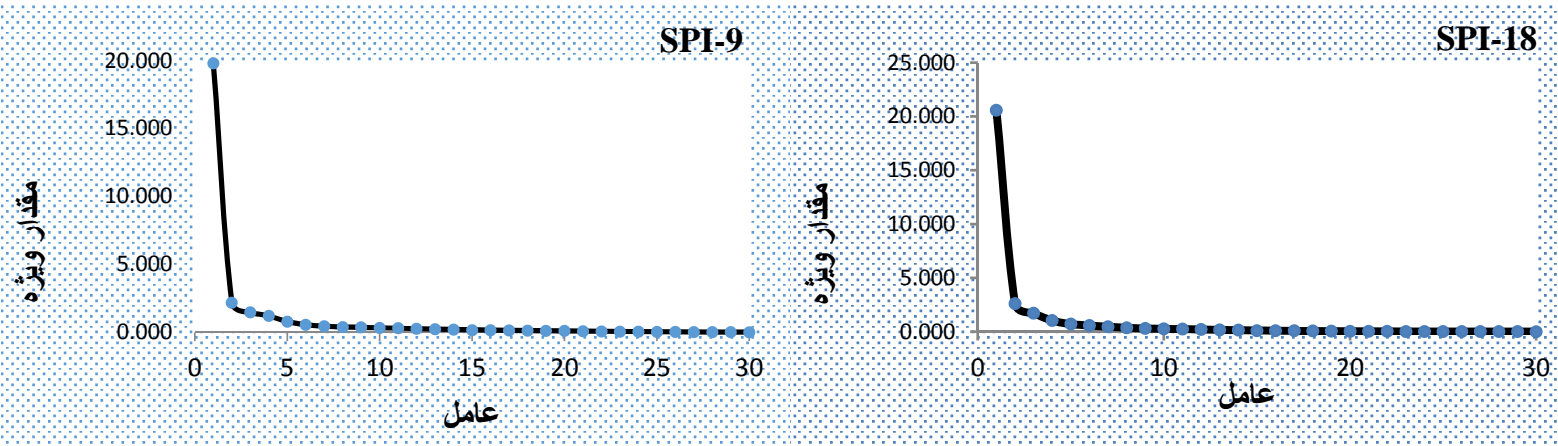

SPI 24
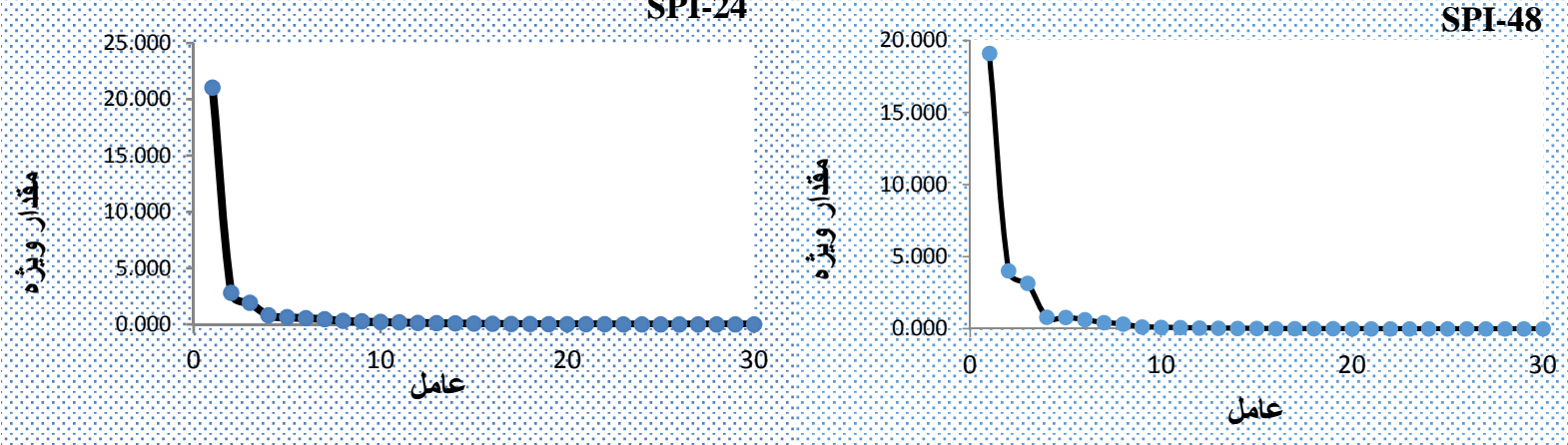

شكل Y) سنكريزه مقياسهاى 9 تا K N ماهه شاخص خشكسالى 
ارتباط الكوهاى ييوند از دور با خشكسالى؛ مطالعه موردى حوضه قرهقوم 109

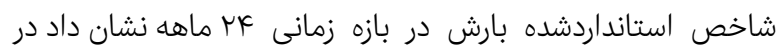

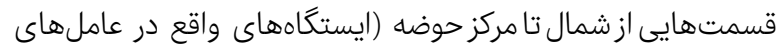
اول و دوم) شرايط خشكسالى از نوع شديد تا بسيار شديد و در عامل

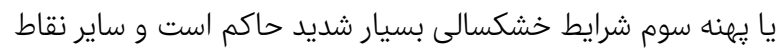
حوضه از شرايط مطلوبترى برخوردار است (شكل ه ه).

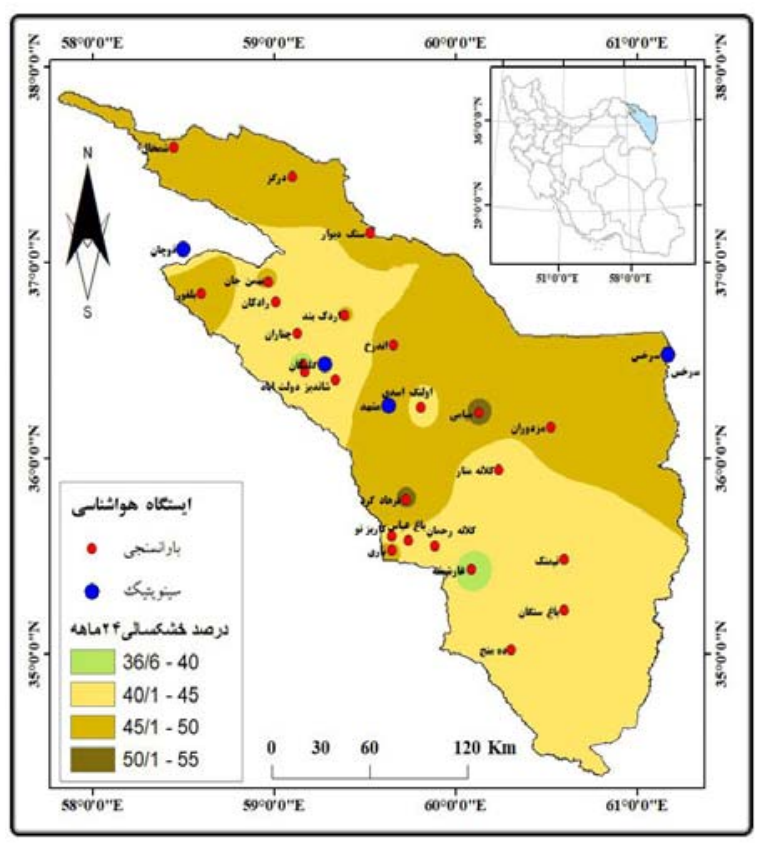

شكل 0) نقشه درصد شاخص خشكسالى MF ماهه

شاخص استانداردشده بارش در بازه زمانى KN ماهه نشان داد شدت

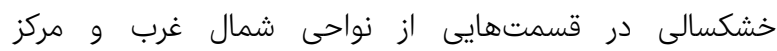

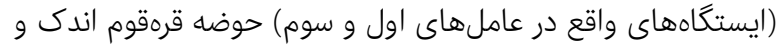
در ساير نقاط حوضه خشكسالى شديد تا بسيار شديد حاكم است ورها

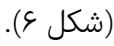

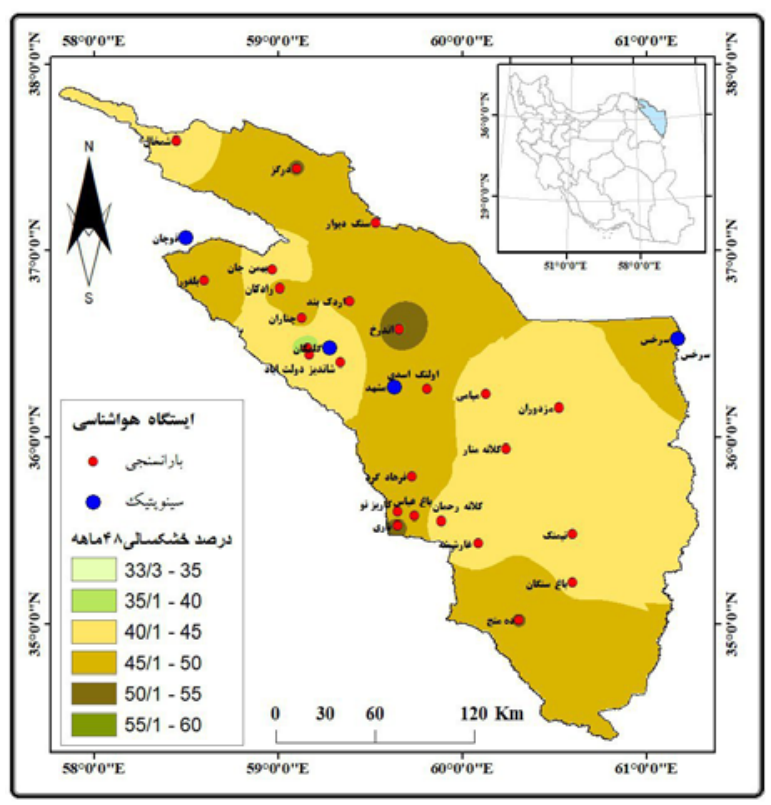

شكل 7) نقشه درصد شاخص خشكسالى KN ماهه

Geographical Researches Quarterly Journal
نقشههاى درصد خشكسالى نشان داد كه شدت خشكسالى در

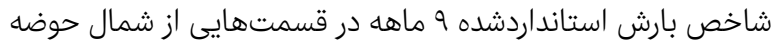

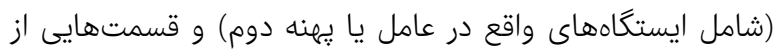

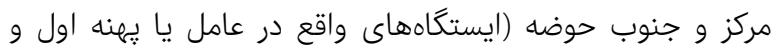

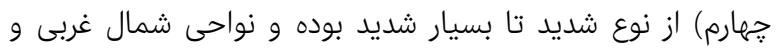

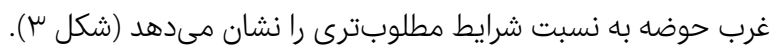

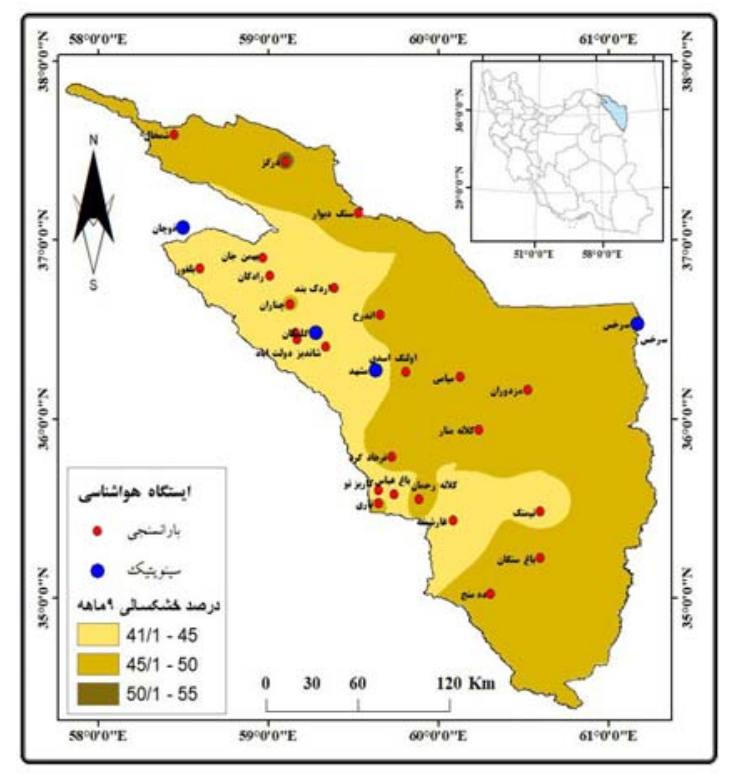

شكل س) نقشه درصد شاخص خشكسالى 9 ماهه

شاخص استانداردشده بارش در بازه زمانى ل ل ماهه نشان داد بهجز

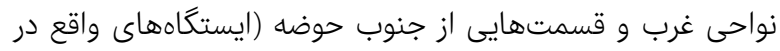

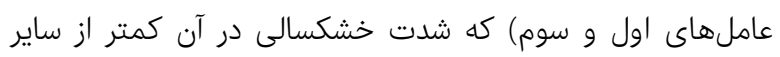

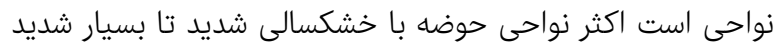

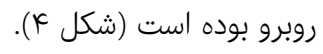

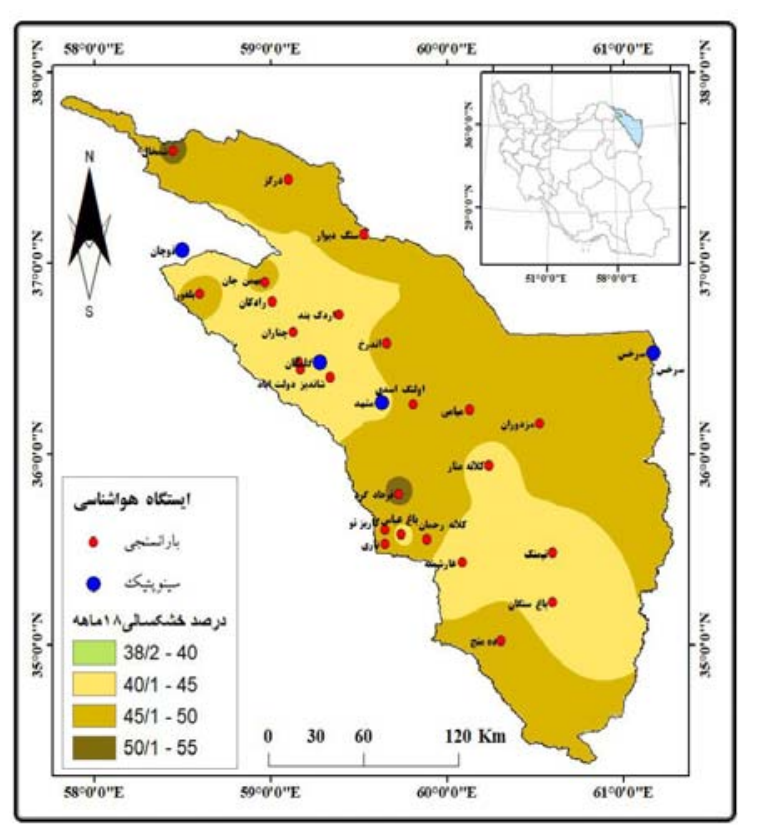

شكل ع) نقشه درصد شاخص خشكسالى 1/ ماهه

Volume 34, Issue 2, Spring 2019 
در منطقه r افريقا-هند در سطح ه9\% معنىدار و با شاخص نوسان

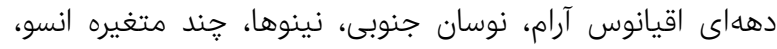

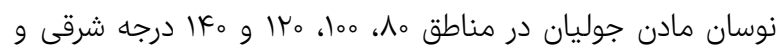

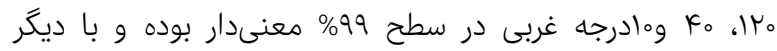

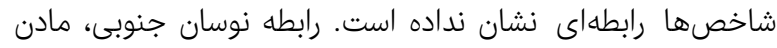

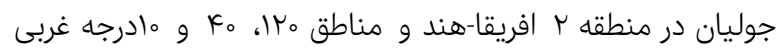
معكوس معنى دار بوده است.

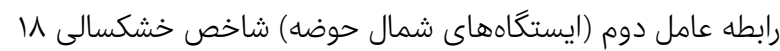
ماهه با شاخص اقيانوس آرام و شمال آمريكا، نوسان اطلس شمالى إنى

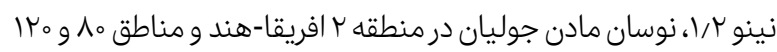

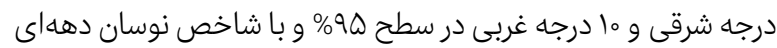

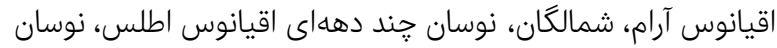

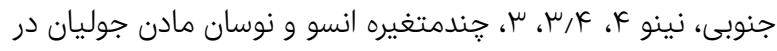

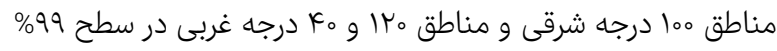

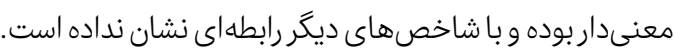

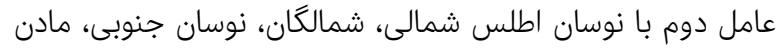

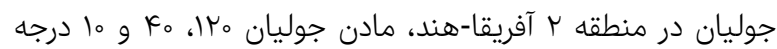

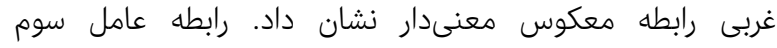

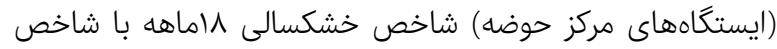

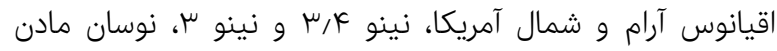

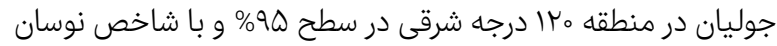

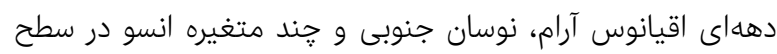
99 99 معنى دار بوده و با ديكر شاخصها رابطهاى نشان ندان نداده است.

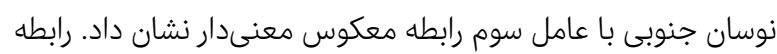

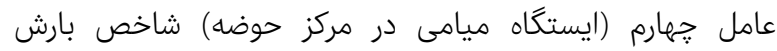

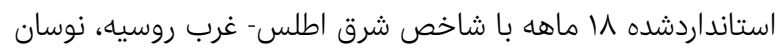

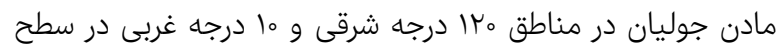

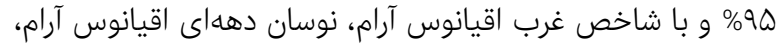

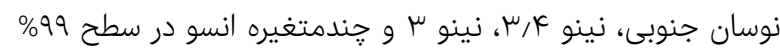

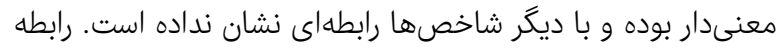

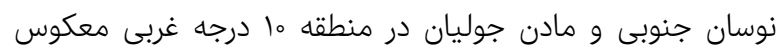

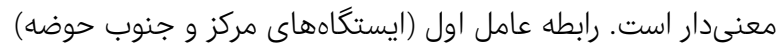

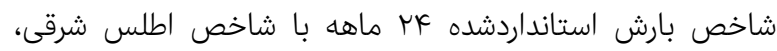

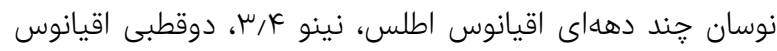

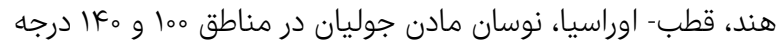
شرقى در سطح هو\% و با نوسان دههاي اقيانوس آرام، آرام شرقى،

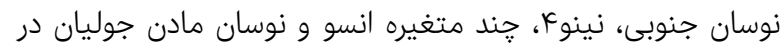

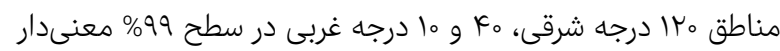

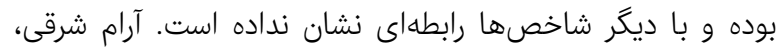

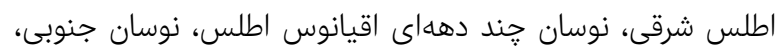

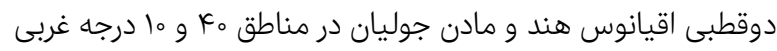

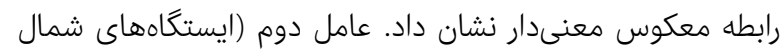

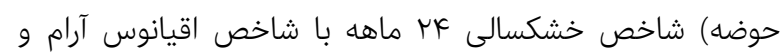

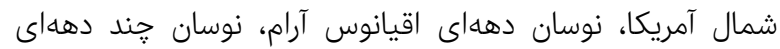

ارتباط بين شاخص بارش استانداردشده با شاخصهاى ييوند از دور

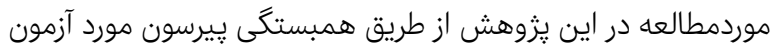

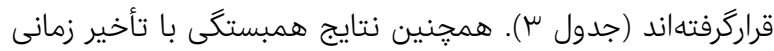

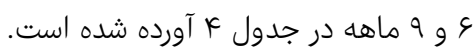
با انجام تحليل عاملى براى هر يك از شاخصهاى خشكسالى وماهـه

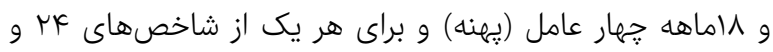

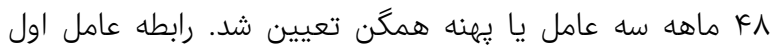

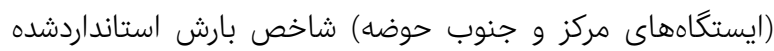

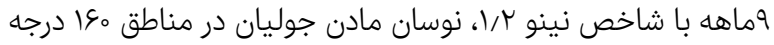

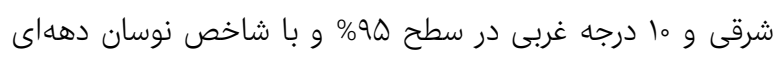

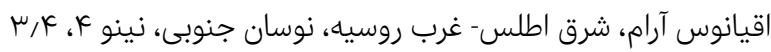

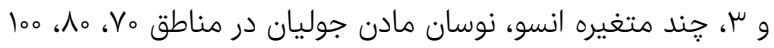

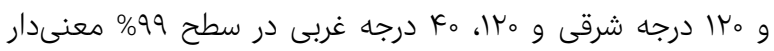
بوده و با ديكر شاخصها رابطهاى نشان نداده است.

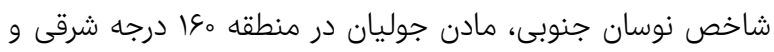

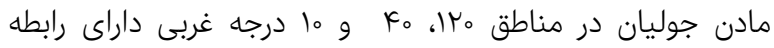

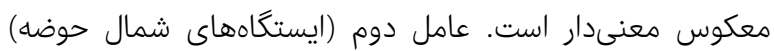

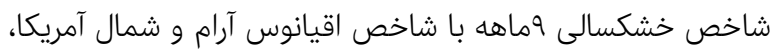

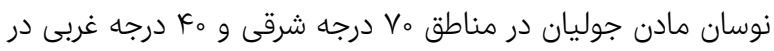

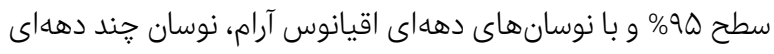

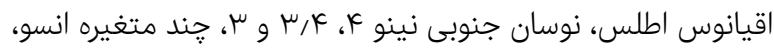

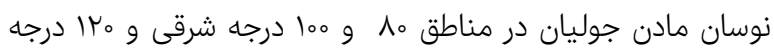
غربى در سطح 99\% رابطه معنىدار داشته و با ديكر شاخصها رابطهاي نشان نداده است.

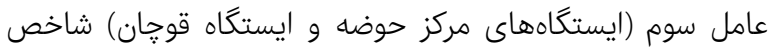

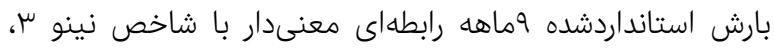

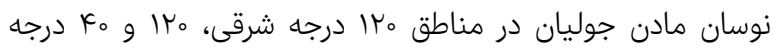

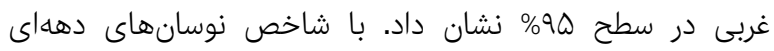

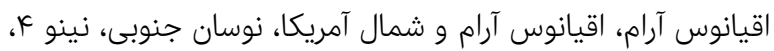

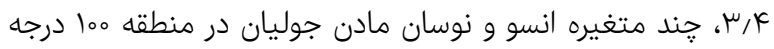

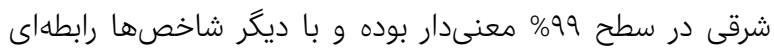

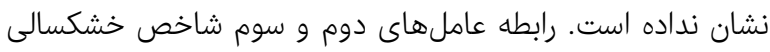

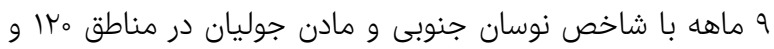

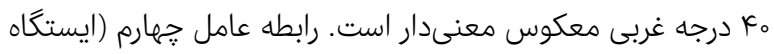

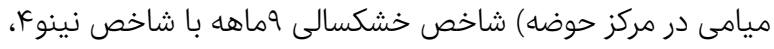

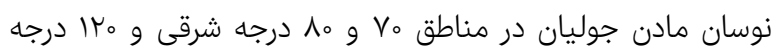

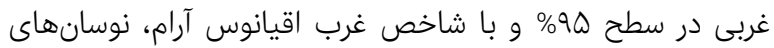

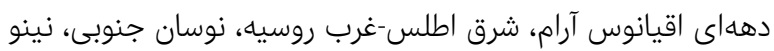

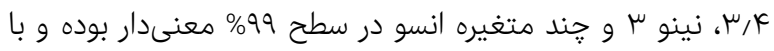
شاخصهاى ديكر رابطهاى نشان نداده است.

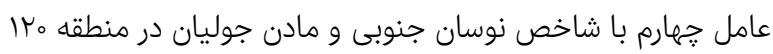

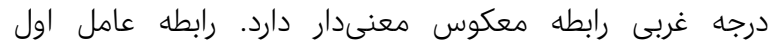

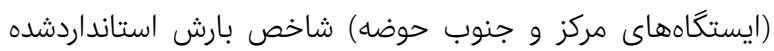
1اماهه با شاخص شرق اطلس- غرب روسيه و نوسان مادن جوليان 


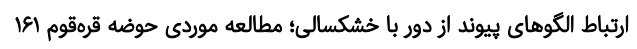

دههاى اقيانوس آرام، جند متغيره انسو و قطب- اوراسيا در سطح

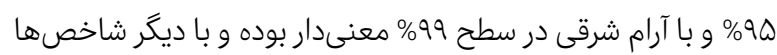

اقيانوس اطلس و جند متغيره انسو در سطح ه9\% و با آرام شرقى

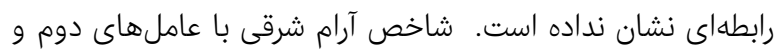

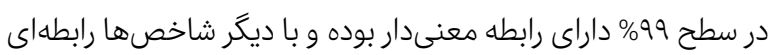
نشان نداده است. رابطه عامل سوم (ايستخاه ميامى در مركز حوضها سوم رابطه معكوس معنىدار نشان داد.

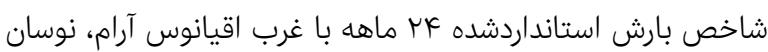

جدول س) ارتباط بين ميانكين عاملهاى خشكسالى و شاخصهاى مختلف بِيوند از دور

\begin{tabular}{|c|c|c|c|c|c|c|c|c|c|c|c|c|c|c|}
\hline r ماد & w & $\infty$ & מס & 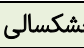 & شاخص & & v & ح & & & ज & c & & شاخص \\
\hline عامل & عامل & عامل & عامل & عامل & عامل & عامل & عامل & عامل & عامل & عامل & عامل & عامل & عامل & \\
\hline سوم & دوم & اول & سوم & دوم & اول & جهارم & سوم & دوم & اول & جهارم & سوم & دوم & اول & \\
\hline \% & $\% 7 \mathrm{TA}$ & $\circ / 111$ & $\circ / I r \Lambda$ & $\%{ }^{\mu} 7$ & $\circ \% \varepsilon \wedge$ & $" \circ / 17 \mu$ & $\% \propto \varepsilon V$ & \%थ। & $\% \wedge \mu$ & $\circ / 1 \varepsilon 0$ & $\% 001$ & $\%{ }^{\mu} \mathrm{V}$ & $\% \% \mu$ & \\
\hline$\% \propto \varepsilon \varepsilon$ & $-0 / 011$ & 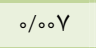 & \% & $\circ / / r_{0}$ & $\% \wedge 10$ & $\% \mu \varepsilon$ & ://w & $\circ / 1 \varepsilon 0$ & $\%$ & $\% 79$ & "ס/rrT & :/ & $\% .97$ & ش شمال امريكا \\
\hline$-0 \%$ \% & $-\% / \%$ & "o/TVY & $\circ / \| \varepsilon$ & ०/1 & $\sigma_{0} / \mu \mu \sigma$ & $" 0 /$ MIM & $" 0 / 790$ & $" \circ / T 9 V$ & $0 / \varepsilon 7 \varepsilon$ & "o/rOV & $" \circ / T \wedge \varepsilon$ & "o/TYY & "o/દાV & ك دهائ القيا \\
\hline$-0 / 0 \varepsilon r$ & ०\% & $\%$ $/ 9 \mu$ &.$/ .91$ & $-0 / 019$ & o $/ \varepsilon V$ & $\%{ }^{\mu} 7$ & $-0 / 0 r \Lambda$ & $\therefore / / \mu \wedge$ & $-0 \% 7 \pi$ & $\%$ ० & $\% \circ \Lambda$ & $-\circ / 0 \varepsilon 9$ & $-0 / 00 V$ & طلس شمالى \\
\hline$"-0 / Y 7 \varepsilon$ & $=0 / Y N 1$ & $"-0 / T V I$ & $"-0 / Y Y \varepsilon$ & $=-0 / T 7 \varepsilon$ & "-o/TOY & $\% \nvdash \varepsilon$ & \% YY & $\% \% 7$ & $\% \mu r$ & $\% \% \varepsilon$ & $\%$ \%Y & $\% Y 7$ & \% & \\
\hline$-0 / 0 \varepsilon Y$ & $-\% / \wedge 9$ &.$/ 097$ & \%^^。 & $\% / 00 Y$ & $\circ / 1.4$ & $\circ / 119$ & \%or & $\% \%$ & $\circ / \mid \varepsilon 1$ & "o/IV^ & \% \& & $\% \propto \wedge \varepsilon$ & $" \circ / 177$ & اطلس و غرب \\
\hline$-0 / 117$ & $\%$ \% r & $\because / 10 \mathrm{r}$ & $\%$ \% & $-\% \odot \Delta^{\circ}$ & 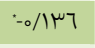 & $\%$ & - & $\%$ \% & $-0 / 001$ & $\% 001$ & $-0 / \mu_{0}$ & $-0 / \% \mu \mu$ & $-0 / 00 \wedge$ & 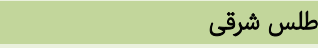 \\
\hline$-0 / 0 \varepsilon 9$ & $-0 / 0 \%$ & $-0 / 010$ & $-0 / 01 V$ & $-\% \%$ & $-0 / 01 \mu$ & $-0 / 009$ & $-0 / 09 \varepsilon$ & $"-0 / 170$ & $-0 / 104$ & $-0 / 099$ & $-0 / 019$ & $-\% / \vee \gamma^{\mu}$ & $-0 / 00 \varepsilon$ & \\
\hline$-0 / 0 Y r$ & $" 0 / 4 \circ 9$ & $"-0 / T \varepsilon \varepsilon$ & $-0 / 09 \mu$ & $\circ / 1 \varepsilon 7$ & $\therefore / / \varepsilon 0$ & $-0 / 019$ & 94 & " & $-\circ / \% \varepsilon$ & $-0 / 0 \mu^{\mu}$ & $\% V r$ & $\% / 10 \mathrm{~V}$ & $-0 \% 0 \mathrm{~K}$ & ان קند دههاى \\
\hline$-0 / 011$ & $-0 / 009$ & $\% 009$ & $-0 / 019$ & $-\% / 4 \mu$ & $-0 / \propto \varepsilon Y$ & $\circ \circ 0 \varepsilon$ & $-0 / 01 \mu$ & $\% / 009$ & - & $\% 009$ & $-0 / 0 Y 7$ & $-\% \propto \varepsilon 9$ & $-0 / .01$ & \\
\hline$\%$ \% A & $-0 \%$ & "-o/YIK & $-0 / 107$ & $-\circ / 0 \wedge 7$ & $"-0 / T \varepsilon \varepsilon$ & "-o/M1O & $"-0 / I V 。$ & "-o/TrY & $"-0 /$ ror & $"-0 / Y M I$ & $"-0 / 4 \wedge 9$ & $=0 / M I Y$ & "- & \\
\hline$-0 / 090$ & $-\% / \% \gamma_{0}$ & \% & $-0 \% \mu \mathrm{V}$ & $\% Y Y$ & $" \circ / 170$ & ००マ৭ & $\% \wedge \vee V$ & $" 0 / Y Y Y$ & $" \circ / \mu \varepsilon_{0}$ & o/lrm & $" 0 / \uparrow \wedge \uparrow$ & 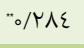 & ${ }_{0} / \varepsilon_{0} 0$ & \\
\hline$-0 / 09 V$ & $-0 / 099$ & $\% \% 99$ & $-0 / 010$ & orr & $\cdot / / \varepsilon \mu$ & $" \circ / 10 \mu$ & $\cdot / / \varepsilon r$ & "o/rro & $\left.{ }^{\circ} / \mu\right\urcorner \varepsilon$ & " & "o/rro & $\circ / / 77$ & "o/rur & \\
\hline$-0 / 0 \wedge 1$ & $\because / / r q$ & $-0 / 04 q$ & $-0 \%$ & $\% / 11$ & $\% \wedge \varepsilon$ & o/ & $\cdot / 11 \Lambda$ & $" 0 / T Y q$ & "o/ YYR & $" \circ / 1$ & ס/NKr & $0 / 190$ & "o/TrE & \\
\hline 。 & $=0 / 1 T_{0}$ & $\% \% \varepsilon$ & $\%$ \% & \% & $\% १_{0}$ & 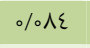 & $\% 70$ & $\because / 1 \varepsilon 7$ & $" 0 / 1 \varepsilon 9$ & o/lor & $\% / 1 \Lambda$ & O/OH & $\circ / 110$ & \\
\hline$\% \% 07$ & $-0 / \%$ Y & " & ://rT & 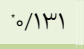 & 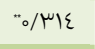 & ${ }^{\circ} / \mathcal{Y} 7_{0}$ & $" \circ / Y Y T$ & " & "o/ $\vee \varepsilon$ & $\mu_{\circ} / \mu_{\circ}$ & "o/ruOV & "о/rrq & $" \circ / \varepsilon १ \varepsilon$ & متغيره انسو \\
\hline$-\circ / \circ 0 \varepsilon$ & $-0 \% \mu r$ & $-0 / 0 \varepsilon \Lambda$ & $-0 \% 70$ & $-\% / 990$ & $\therefore /|\mu|$ & \%o०r & $-\circ / \circ \wedge Y$ & \% \% & $-0 / 07 V$ & $\%$ & $-0 / 009$ & $\% 71$ & $-0 / 010$ & قطبى اقيانوس هن: \\
\hline$\% \vee \wedge$ & $\% \vee V 1$ & $\circ / 10 \varepsilon$ & $\circ / / \mu \mu$ & \%०ᄉ & ( & $\% \circ 9$ & $\% \varepsilon V$ & ०० $\left.{ }^{\mu}\right\rceil$ & $\% \wedge \Lambda$ & $-\circ / \circ \varepsilon 1$ & $-0 / 001$ & $-0 / 090$ & $-0 / 00 r$ & ب-اوراسيا \\
\hline$\% \mu r$ & $\% 0 r$ & \% $\%\}$ & of & ००८દ & $r$ & i & $\wedge_{0}$ & .00 & $-\circ / 04 \Lambda$ & $\wedge$ & $-\% / 000$ & $\% / 1 \Lambda$ & $\mu$ & 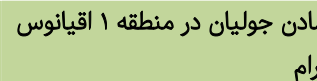 \\
\hline
\end{tabular}

مادن جوليان در منطقه r افريقا-

نمايه فاز نوسان مادن جوليان

نمايه شدت و دامنه نوسان مادن

جوليان مادن جوليان در منطقه هrدرجه

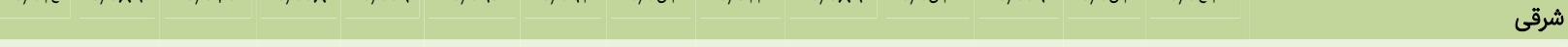
مادن جوليان در منطقه

\begin{tabular}{|c|c|c|c|c|c|c|c|c|c|c|c|c|c|c|}
\hline$-0 / 07 \Lambda$ & $-0 / 07 \varepsilon$ & $-0 / \% q$ & $-0 / 019$ & $\% \% Y 7$ & $\% 7 V$ & $\% \varepsilon_{0}$ & $\%$ & $\circ / 1 \varepsilon O$ & "o//£r & $\circ / \| \varepsilon$ & $\circ / 11$ & $" 0 / 171$ & "०/191 & ماد درجه جوليان در منطقه \\
\hline$-\% \% 70$ & $\theta_{0} /|\mu|$ & $\%$ & $\% / 0 \%$ & $\% \varepsilon r$ & $\circ / / \varepsilon_{0}$ & $\% .97$ & $\% 97$ & "o/ $/ Y 7$ & " o/rאO & ./III & o & $" \circ / 107$ & "o/MIY & ما دادجه شوليان در منطقه \\
\hline $1.4 q$ & $"-0 / 170$ & $0 / 091$ & $0 / 017$ & $\%$ \% & $0 / 177$ & 喟 & $\circ / 110$ & $\circ / 109$ & "o/rOA & $\% \vee \vee 7$ & י/R & $\circ / 11$ & $" \circ / I V r$ & مادادرجه شوليان در منطقه \\
\hline$-0 / 00 Y$ & A & $\circ / 1.9$. & \% Orr & $\% \mu_{0}$ & س س & $0 / 107$ & \%०१६ & $\% \wedge \mu$ & $" \circ / 190$ & $\%$ & $\% 77$ & .017 & $\% 77$ & مادراد جوليان در منطقه \\
\hline$\%$ \% r & $-0 / 0 \mu_{0}$ & $\% 7 \varepsilon$ & $\% / 1 \varepsilon$ & $-0 / 007$ & \%/ol & $\%$ \% & $-0 / 014$ & $-0 / 001$ & $-0 / 00 \mu$ & $-0 / 090$ & $-0 / 0 \varepsilon \mu$ & $-0 / 10 \wedge$ & $=0 / I I r$ & مادادرجه شوليان در منطقه \\
\hline$\% 09$ & $\% \wedge \wedge r$ & $-0 / 010$ & $-0 / 00 Y$ & - & $-0 / 10 r$ & - & $-0 / 07 V$ & $=0 / 104$ & $"-0 / I V Y$ & $\therefore / / K \varepsilon$ & $=0 / 110$ & $=-0 / 109$ & $"-0 / T \circ \varepsilon$ & مادادرجه غوليان در منطقه \\
\hline$\% 01$ & o/lEr & $-0 / \% 70$ & $-0 / 00 Y$ & $-0 / \%$ T & $"=-1 / 109$ & $-0 / 111$ & $-\circ / 1 \circ \wedge$ & $"=0 / 1 Y O$ & $"-0 / r \varepsilon V$ & $-0 / 1 \circ \Lambda$ & - $=0 / 1 \mu r$ & $\therefore / / \varepsilon O$ & $"=0 / T \cdot O$ & مادن جوليان در منطقه غرجيى \\
\hline$\%$ \% ג & $" \circ / 17 \circ$ & $-0 / 09 \varepsilon$ & $-0 / 017$ & $-0 / \& \varepsilon 1$ & $"=0 / 100$ & $\therefore / / r_{0}$ & $-0 / 111$ & $\therefore / / \pi V$ & ג א & $-0 / 001$ & $-0 / / 0 \varepsilon$ & $-\% / \vee \vee ৭$ & $-0 /|\mu|$ & مادرجه غوبيى دربان در منطقه \\
\hline
\end{tabular}


rاء مونا فلاحزاده و همكاران

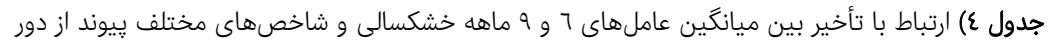

\begin{tabular}{|c|c|c|c|c|c|c|c|c|}
\hline \multicolumn{4}{|c|}{ شاخص بارش استاندارد شده } & \multicolumn{4}{|c|}{ شاخص خشكسالى با تأخير 7 ماهه } & \multirow{3}{*}{ شاخم شاخص خشكسالى با تأخير 9 ماهه } \\
\hline عامل & عامل & عامل & عامل & عامل & 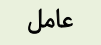 & عامل & عامل & \\
\hline 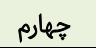 & 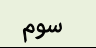 & 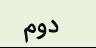 & اول اول & جهارم & 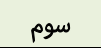 & 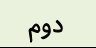 & اول اول & \\
\hline$\% / 990$ & $-0 / 00 \varepsilon$ & $-\circ / 0 \mu_{0}$ & $-0 / 0 \curlyvee \varepsilon$ & $\% 7 r$ & $\% \mu \wedge$ & $\% / \mathrm{V}$ & $\% / 009$ & غرب آرام \\
\hline$-\circ / \circ \varepsilon \mu$ & $-0 \% 000$ & $\%$ & $-\% \vee \vee q$ & $-0 / 04 q$ & $\% \propto\{7$ & $-o / \% \varepsilon$ & $-0 / .00$ & اقيانوس آرام و شمال امريكا \\
\hline$\%$ \%O & $" \circ / 19 \varepsilon$ & $\% \mu r$ & $" 0 / Y \circ V$ & $" \circ / I V Y$ & $\% 07$ & $\% 94$ & $\circ / \Lambda \Lambda "$ & نوسانهاى دههاى اقيانوس آرام \\
\hline$\% \wedge \mu$ & $\% \mu \mu$ & \% & $\circ / 1 \mu$ & $-\%$ \% & $-0 / \% Y$ & $\% 71$ & $\%$ & نوسان اطلس شمالى \\
\hline$"-0 /$ NIR & $"-0 / Y \varepsilon O$ & $"-0 / r \varepsilon q$ & $"-\circ / \Gamma \varepsilon \mu$ & $"-0 / Y \varepsilon V$ & $"-\circ / R \mu V$ & $"-0 / R \varepsilon \varepsilon$ & $"-0 / \Gamma \varepsilon \Lambda$ & 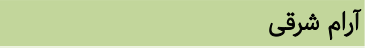 \\
\hline \%or & $" 0 / 107$ & " & $" \circ / 1 \varepsilon \Lambda$ & $" \circ / \wedge \varepsilon \Lambda$ & "o/lर。 & $\%$ & "O/IMI & شرق اطلس و غرب روسيه \\
\hline$-0 / 0 \vee 7$ & $\% \circ \%$ & $\%$ & $-\circ / .9 \mu$ & $\% 77$ &.$/ 1.7$ & $-0 \%$ & $-0 / . \mu 7$ & اطلس شرقى \\
\hline$\% / 097$ & $-0 / 010$ & $-0 / \% \uparrow$ & $\% 000$ & $-0 \% 0 \mathrm{r}$ & $-0 \% \vee 7$ & $-0 \%$ \% & $\circ \%$ & شمالگان \\
\hline$\because / / 1 \mu 7$ &.$- / .0 V$ & $-0 / \odot \varepsilon 1$ & "-o/ ROQ & $-0 / 01 \mu$ & $\% \mu^{\mu}$ & $*-0 / 1 \mu q$ & "-o/rYA & نوسان حند دههاى اقيانوس اطلس \\
\hline$-0 / 11 \wedge$ & -o/\%O & $\% \circ \wedge$ & $\% \circ \mu^{\mu}$ & $-\circ / 01 \mu$ & $\% \mu \varepsilon$ & $\%$ \% r & \%४r & اسكانديناوى \\
\hline$\therefore / 111$ & $"-0 / \mu 77$ & $"-0 /$ NIR & $n_{-\circ / \mu V Y}$ & $"-0 / \varepsilon_{0} Y$ & $"-0 / Y 70$ & $"-0 / \mu \varepsilon\rceil$ & "-o/乏)V & نوسان جنوبى \\
\hline$\% \circ 1$ & $" 0 / \varepsilon \varepsilon 7$ & $" 0 / \mu I V$ & $" 0 / \varepsilon / \varepsilon$ & $" \circ / \varepsilon \mu q$ & "。/YAT & $" \circ / \mu 79$ & "o/rAV & 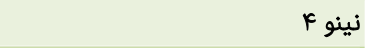 \\
\hline$\% \wedge \wedge$ & $" \circ / \varepsilon \mu r$ & $" \circ / \uparrow \wedge O$ & ${ }^{\circ} \circ / \varepsilon_{\circ}$ & $" 0 / \varepsilon M$ & "o/ro。 & $" \circ / \mu 77$ & $" \circ / \% \wedge O$ & نينو أر \\
\hline$": 117$ & $" 0 / \mu \mid \Lambda$ & $" \circ / \backslash A Y$ & "o/ TO० & $"$ "०/Tqع & $" 0 / 101$ & $" \circ / T r Y$ & $" 0 / Y \varepsilon Y$ & 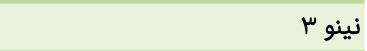 \\
\hline$" 0 / 1 m q$ & $" 0 / \backslash \wedge \vee$ & $\% \vee 1$ & $\circ / 1 . \mu$ & $" \circ / / \varepsilon 9$ & $\% \mathrm{VI}$ & $\%$ & : & 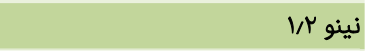 \\
\hline$" \circ / I V Y$ & $" \circ / \varepsilon 0_{0}$ & $" 0 / Y \mu 1$ & $" \circ / \mu \wedge \lambda$ & $" \circ / \varepsilon \varepsilon \mu$ & 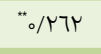 & $" \circ / \% 00$ & $" 0 / \varepsilon \mid \gamma$ & جند متغيره انسو \\
\hline$-0 \% \mu V$ & $\% 990$ & $\% \psi^{\circ}$ & o/lr & "०/1mq & $-0 / 01 \varepsilon$ & " $/ / \mu \mu$ & $\%$ \% & دو قطبى اقيانوس هند \\
\hline o/l1r & $\% \wedge \Lambda$ & $\% \wedge 7$ & $\circ / 11 \mathrm{~V}$ & $\% 71$ & $\% 91$ & $\% \wedge 7$ & $\circ / 110$ & قطب- اوراسيا \\
\hline$\% \varepsilon \mu$ & $\%$ \% & $-0 / 04 q$ & $-\%$ rO & $-\circ / 0 \varepsilon_{0}$ & $-0 \% 0$ & $-0 / 07 \mu$ & $-0 \% 7 r$ & مادن جوليان در منطقه I اقيانوس آرام \\
\hline$\% \mu r$ & $\%$ & $\% \vee \vee q$ & $\%$ \% & $\%$ \% & $\% 7 \Lambda$ & $\% 7 \pi$ & $\%$ \% & مادن جوليان در منطقه r افريقا-هند \\
\hline$\% \wedge \Lambda$ & $\% \vee q$ & $\circ / 14 q$ & $\% 97$ & $\% \vee V$ & $\%$ & $\% .97$ & $\circ / 1.4$ & نمايه فاز نوسان مادن جوليان \\
\hline$\% \% r$ & $-0 / 009$ & $\%$ $\%$ & $\% 19$ & $-0 / 009$ & $\% 0 r$ & $\%$ \% & $\% 7 \%$ & نمايه شدت و دامنه مادن جوليان \\
\hline$\%$ \% & $" \circ / T V 7$ & $" 0 /$ MIT & $" \circ / 194$ & $" \circ / T \mu_{0}$ & $" 0 / 197$ & $" \circ / 171$ & $" \circ / / \Lambda 1$ & مادن جوليان در منطقهـ درجه شرقى \\
\hline "o/lr & $" \circ / \mu \wedge \mu$ & 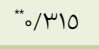 & "。/ror & 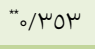 & $" \circ / \sim q \mu$ & $" 0 / \mu \circ r$ & $" \circ / \mu V_{0}$ & مادن جوليان در منطقهه درجه شرقى \\
\hline$\because / N Y$ & $" \circ / \mu \vee\urcorner$ & $" \circ / \mu \mid \Lambda$ & "o/ror & $" \circ / \mu 71$ & $" \circ / \mu_{0} \mu$ & $" \circ / \mu \mid \varepsilon$ & $" \circ / \pi \vee q$ & مادن جوليان در منطقهـ1 درجه شرقى \\
\hline$\circ / 1 \varepsilon O$ & 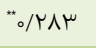 & $" \circ / r \varepsilon \Lambda$ & $" 0 / \mu 10$ & $" 0 / 410$ & "o/rro & $" 0 / T V 7$ & $" \circ / \mu o$ & مادن جوليان در منطقه م-ادرجه شرقى \\
\hline$* / 117$ & $\circ / 110$ & o/IIr & $" \circ / 191$ & $\therefore / 1 \mu 7$ & $\circ / 1 \circ V$ & $" \circ / 170$ & $" 0 / T r \varepsilon$ & مادن جوليان در منطقه •rادرجه شرقى \\
\hline ॰ & $=0 / 1 \mu \mu$ & $-\circ / .90$ & -。/\%o & $-\circ \% 9 \varepsilon$ & $-\circ / \wedge \vee V$ & $-\circ /{ }^{\mu r}$ & $-\circ / \circ \mid \varepsilon$ & مادن جوليان در منطقه \& ادرجه شرقى \\
\hline$-0 / 019$ & $"-0 / \mu_{0}$ & $"-0 / Y \Lambda \Lambda$ & $"-\circ /$ YAV & $"-0 / \mu 17$ & $"-o / T Y 。$ & $"-0 / \Gamma \varepsilon q$ & "-о/ ศq & مادن جوليان در منطقه •غادرجه شرقى \\
\hline$"-$-o/10r & $"-0 / \mu 71$ & $"-0 / \mu_{0} 7$ & "-o// 07 & $"-0 / \mu \varepsilon \varepsilon$ & $"-\circ /$ YAV & $"-0 / \mu_{0} \Lambda$ & $"-\circ / \mu \wedge \varepsilon$ & مادن جوليان در منطقه •rادرجه غربى \\
\hline$\because-\circ / 1 \varepsilon \Lambda$ & $"-0 / \Gamma \varepsilon 1$ & "-o/TIE & $"-\circ / Y \Lambda \Lambda$ & $"-0 / R \varepsilon 7$ & $"-0 / T_{01}$ & $"-0 / Y \varepsilon \Lambda$ & $"-о / \mu \mu \mu$ & مادن جوليان در منطقه Fo درجه غربى \\
\hline$-\circ \% \wedge \Lambda$ & $-0 \%$ Hr & $-\circ / \mu_{\mu} \mathrm{V}$ & $-\circ / 10 \Lambda$ & $-\% / 001$ & $-\circ / \mu^{\mu} \mathrm{V}$ & $-\% / .9 \varepsilon$ & $-0 / / 14 q$ & مادن جوليان در منطقه •ادرجه غربى \\
\hline
\end{tabular}

نشان نداده است. آرام شرقى، نينو س، نينو r/ر، مادن جوليان در

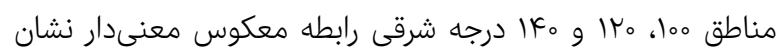

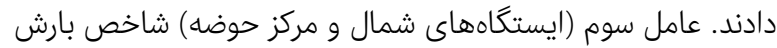

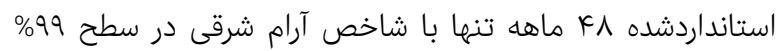
رابطه معكوس معنىدار نشان داد.

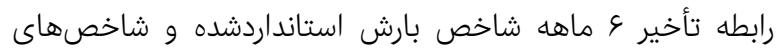

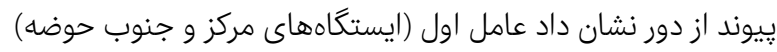

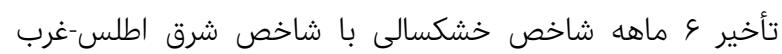

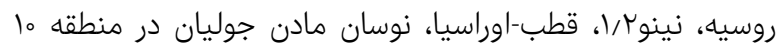

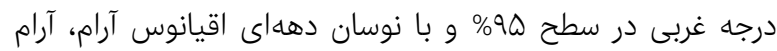

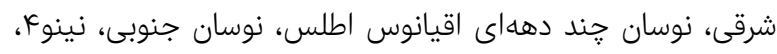

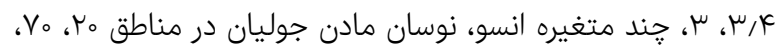

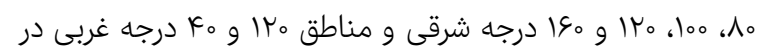

عامل اول (ايستخاههاى مركز و جنوب حوضه) شاخص بارش

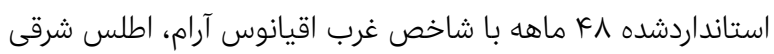
و قطب- اوراسيا در سطح ه9\% و با شاخص نوسان دههاى اقيانوس

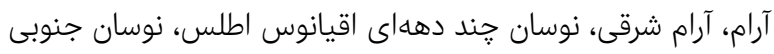

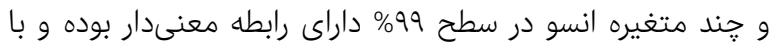

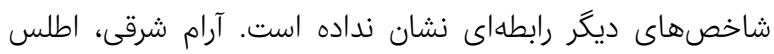

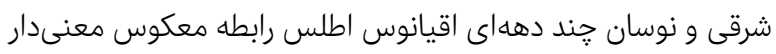

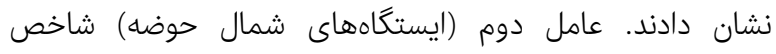

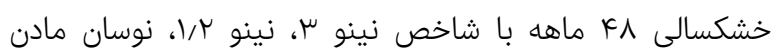

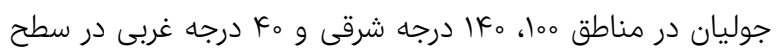

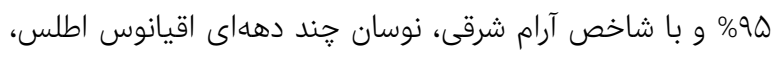

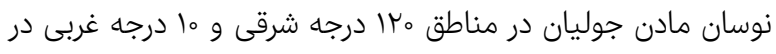

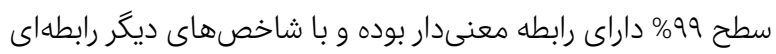




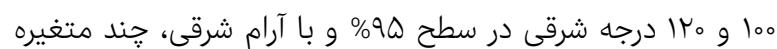

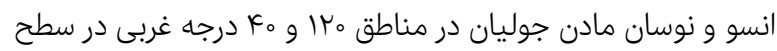

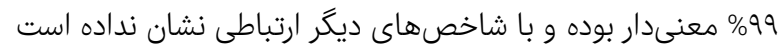

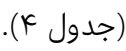

\section{نتيجه}

در حوضه قرهقوم بيشترين سطح معنىدارى مربوط به شاخص جند

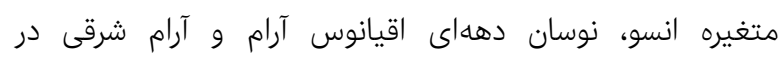

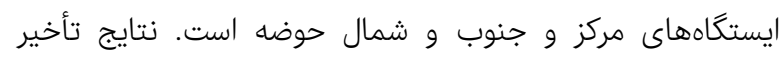

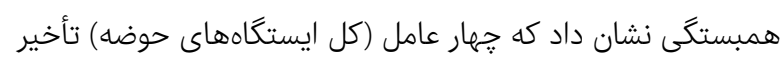

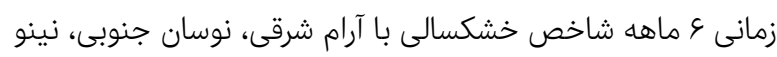

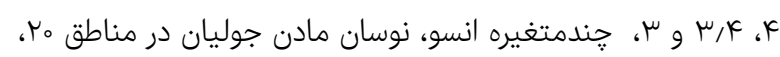

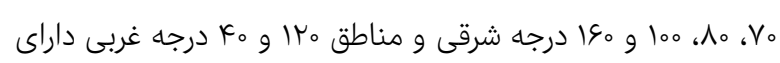

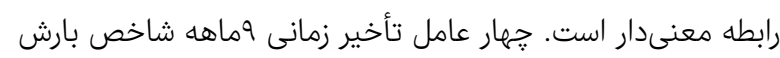

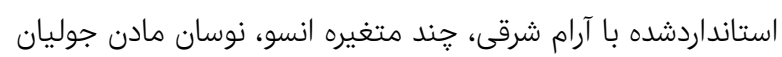

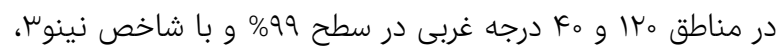

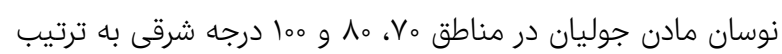
در سطح 99 و ه9\% داراى رابطه معنىدار است.

تشكر و قدردانى: موردى از سوى نويسندكًان گَزارش نشده است.

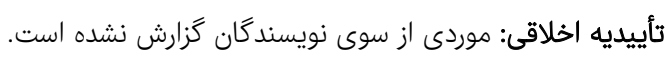

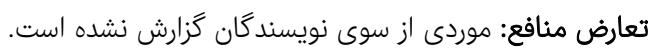

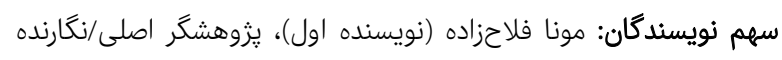

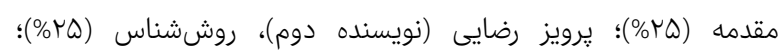

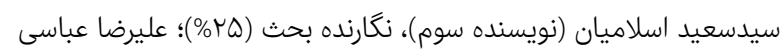

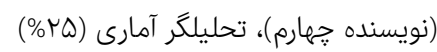

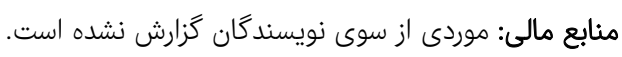

منابع

Ilderumi A, Noori H, Bayat Varkeshi M (2017). Study of Relation between ENSO phenomenon and drought occurrence in Iran. Water and Soil Science. 27(2):143156. [Persian]

Babaiean I, Zarghami M, Kouhi M, Babaiean O, Karimain M, Modirian R (2013). Investigating the behavior of Qara Qom Basin water resources under the conditions of climate change (Case study: Dargaz sub basin). Water and Soil (Agriculture Sciences and industries). 27(5):907-918. [Persian]

Khosravi M (2004). A Survey on the relations of the northern hemisphere large scale circulation patterns with Sistan \& Baluchestan annual droughts. Geography and Development. 2(3):167-188. [Persian]

Khorshiddost A, Rahimi Ghavidel Y, Abaszadeh K (2010). Application of large-scale atmospheric-oceanographic patterns in the analysis of rainfall oscillations (Case study: Ahar station). Geographical Space. 10(29):95-128. [Persian]

Darand M (2015). Drought monitoring in Iran by Palmer Severity Drought Index (PDSI) and correlation with oceanic atmospheric teleconnection patterns. Geographical Researches. 29(4):67-82. [Persian]

Zolfaghari H, Nouri Sameleh Z (2016). Determination of
سطح 99 \% معنى دار بوده و با شاخصهاى ديگر ارتباطى نشان نداده است. عامل دوم (ايستخاههاى شمال و مركز حوضه) تأخير زمانى

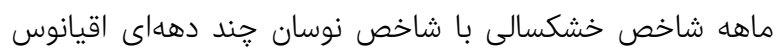
اطلس، دوقطبى اقيانوس هند در سطح ه9\% و خ با آرام شرقى، نوسان

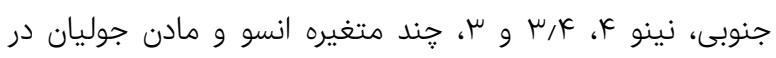

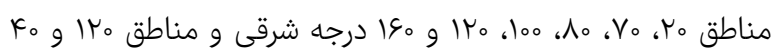

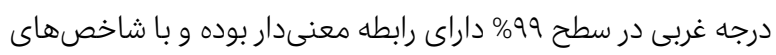

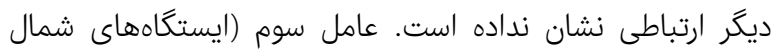

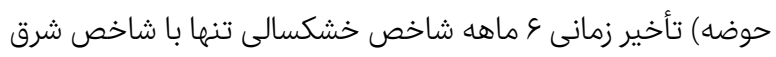
اطلسغرب روسيه در سطح ه9\% و با شاخص آرام شرقى، نوسان

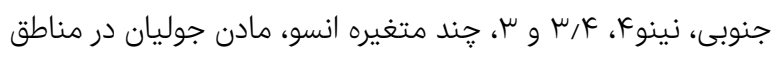

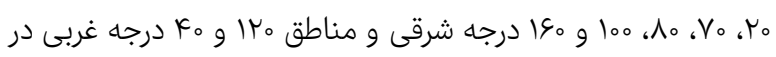

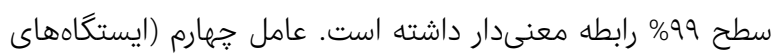

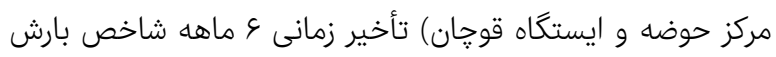

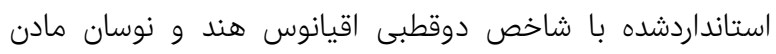

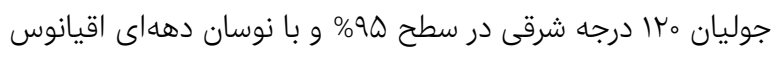

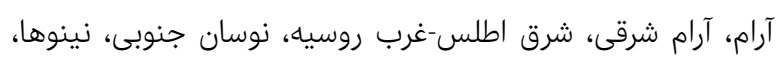

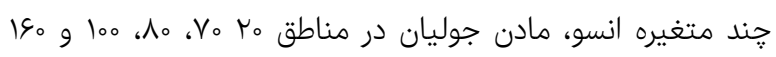

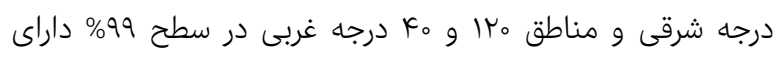

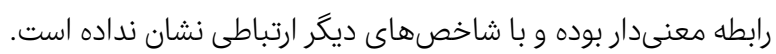

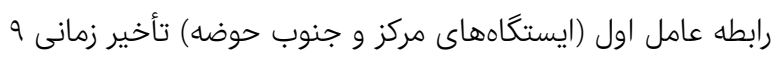
ماهه شاخص خشكسالى با نوسان اطلس شمالى، دوقطبى اقيانوس إنس

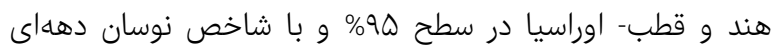

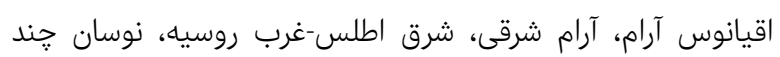

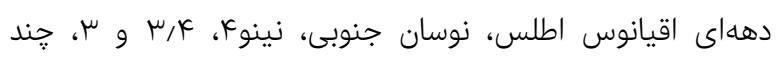

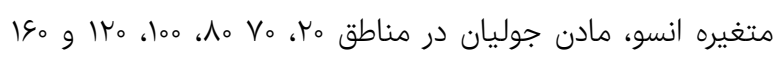

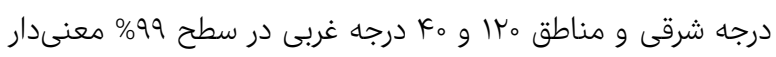

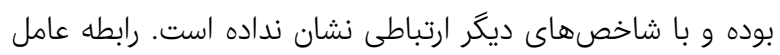

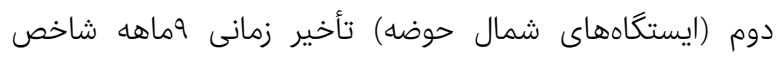
خشكسالى با شرق اطلس- غرب روسيه و نمايه فاز نوسان مادن

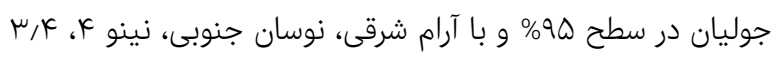

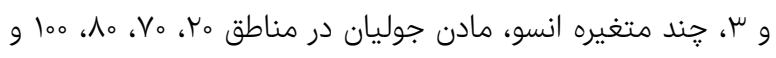

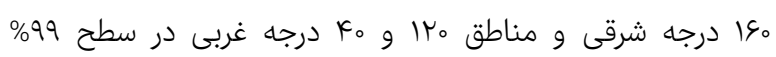

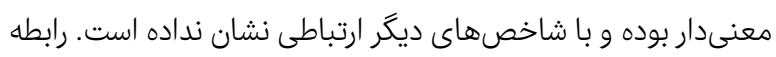
عامل سوم (ايستخاههاى مركز و ايستگگاه قوجان) تأخير زمانى

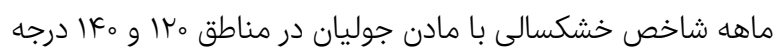

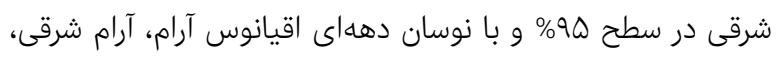

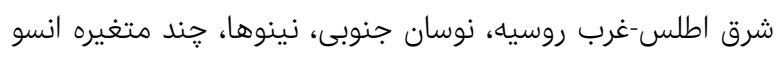

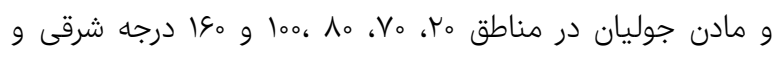

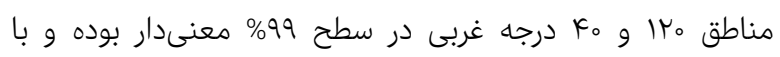

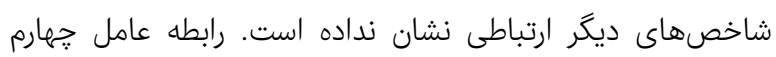

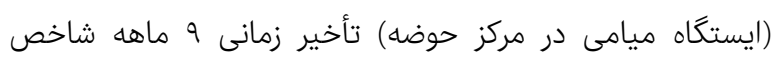

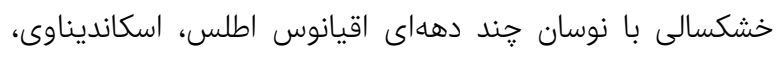

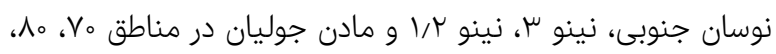


in geography. $4^{\text {th }}$ Edition. Tehran: Ghomes Publications. [Persian]

Nayebi H (2015). Advanced applied statistics with SPSS. $2^{\text {nd }}$ Edition. Tehran: Tehran University Press. [Persian] Asong ZE, Wheatear HS, Bonsal B, Razavi S, Kurkute S (2018). Historical drought patterns over Canada and their teleconnections with large-scale climate signals. Hydrology and Earth System Sciences (HESS). 22:31053124.

Baek SH, Smerdon JE, Coats S, Williams AP, Cook BI, Cook ER, Seager R (2017). Precipitation, temperature, and teleconnection signals across the combined North American, Monsoon Asia, and old world drought Atlases. Climate. 30(18):7141-7145.

Chowdhury MR (2003). The El Nino- Southern Oscillation (ENSO) and seasonal flooding Bangladesh. Theoretical and Applied Climatology. 76(1-2):105-124.

Hendon HH (2003). Indonesian rainfall variability: Impacts of ENSO and local air-sea interaction. Climate. 16(11):1775-1790.

Lee HF, Zhang DD (2011). Relationship between NAO and drought disasters in northwestern China in the last millennium. Arid Environments. 75(11):1114-1115.

Oglesby R, Feng S, Hu Q, Rowe C (2012). The role of the Atlantic multidecadal oscillation on medieval drought in North America synthesizing results from proxy data and climate models. Globaland Planetary Change. 84-85:56-65. Rajagopalan B, Cook E, Lall U, Ray BK (2000). Spatiotemporal variability of ENSO and SST teleconnections to summer drought over the United States during the twentieth century. Climate. 13(24):4244-4255.

Wang H, Kumar A (2015). Assessing the impact of ENSO on drought in the U.S. Southwest with NCEP climate model simulations. Hydrology. 526:30-41.
秘

suitable variables for analysis of droughts in Iran by using CPEI index. spatial analysis of environmental hazards. 3(3):99-114. [Persian]

Sadatinejad J, Alavinia H, Abedi R, Honarbakhsh A, Abdollahi Kh (2016). Frequency analysis of regional meteorological drought in Karun-1 basin of Iran. Watershed Management Research. 6(12):108-117. [Persian]

Shafiei M, Raeini M (2016). Assessment of the impact of meteorological drought and wetness trends on the Parishan lake water level decline. Watershed Management Research. 6(12):118-126. [Persian]

Shafiee M, Ghahraman B (2009). Investigation of place variations of maximum probable 24-hour precipitation in Qara Qom basin. Irrigation and Drainage. 3(2):50-59. [Persian]

Alizadeh A, Erfanian Arghavanian Sarshoory M, Ansari H (2011). Investigating the effect of teleconnection patterns on parameters of precipitation and temperature (Case study: Synoptic station of Mashhad). Irrigation and Drainage. 5(2):176-184. [Persian]

Fatehi Marj A (2015). Study of the relationship between the some climate signals (SOI, NAO, MEI, NINO) and meteorological drought in Kerman province, Iran. Agricultural Meteorology. 3(1):25-39. [Persian]

Ghavidel Rahimi Y, Farajzadehasl M, Kakapoor S (2014). Investigation on North Sea-Caspian teleconnection pattern effect on autumn rainfall fluctuations in west and northwest regions of Iran. 18(49):217-230. [Persian]

Ghavidel Rahimi Y, Hatami Zarneh D, Rezaei M (2014). The role of a teleconnection pattern the far atmosphere of the North Sea-Mazandaran Sea in the changes in the rainfall of the southern shores of the Caspian Sea. Applied Geosciences Research. 13(31):29-46. [Persian] Mahdavi M, Taherkhani M (2012). Application of statistics 